\title{
California Groundwater Units
}

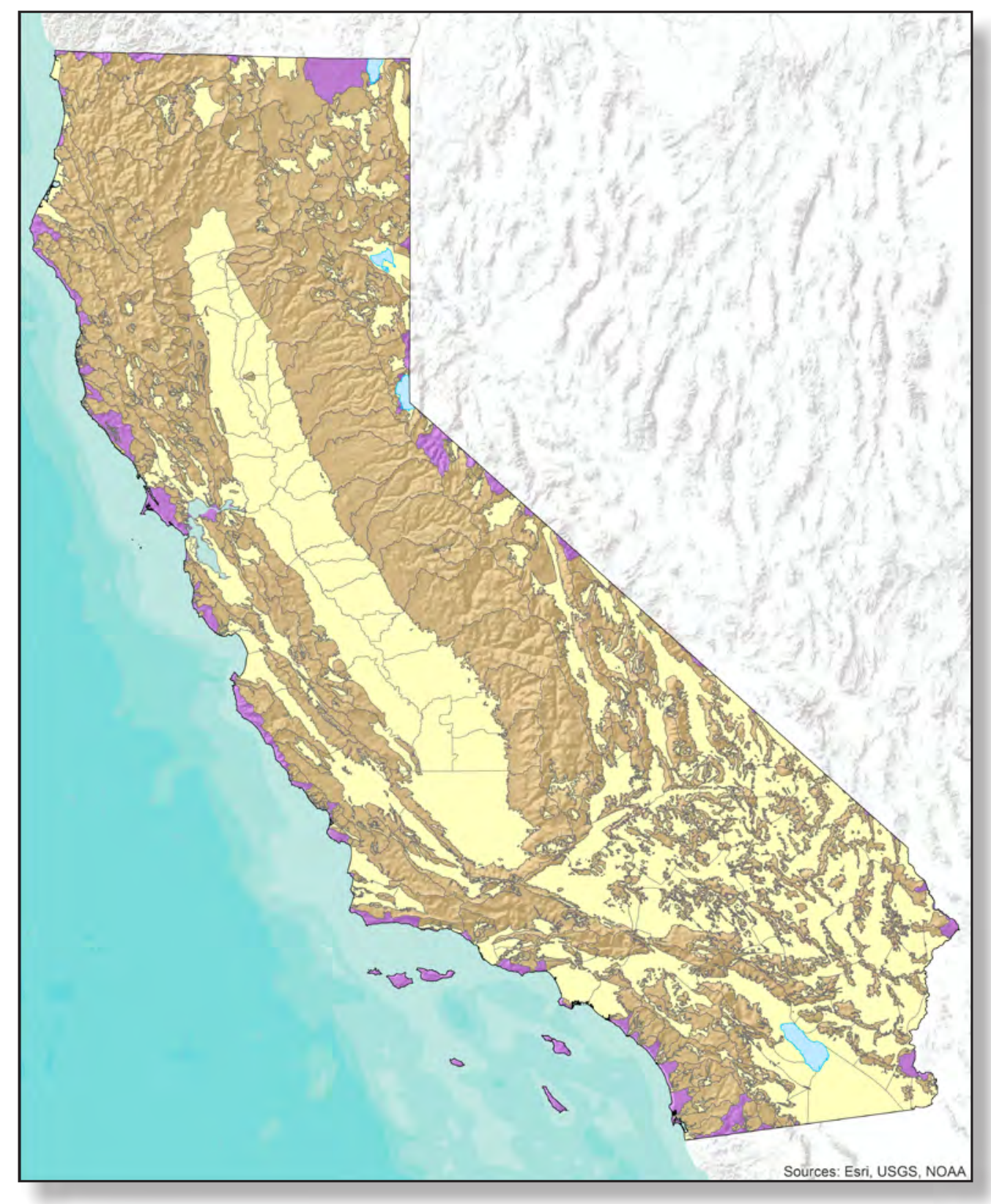

Data Series 796 
Cover. California Groundwater Units. 


\section{California Groundwater Units}

By Tyler D. Johnson and Kenneth Belitz

Data Series 796

U.S. Department of the Interior

U.S. Geological Survey 


\title{
U.S. Department of the Interior SALLY JEWELL, Secretary
}

\section{U.S. Geological Survey Suzette M. Kimball, Acting Director}

\author{
U.S. Geological Survey, Reston, Virginia: 2014
}

For more information on the USGS - the Federal source for science about the Earth, its natural and living resources, natural hazards, and the environment, visit http://www.usgs.gov or call 1-888-ASK-USGS.

For an overview of USGS information products, including maps, imagery, and publications, visit http://WwW.usgs.gov/ pubprod.

To order this and other USGS information products, visit http://store.usgs.gov.

Any use of trade, firm, or product names is for descriptive purposes only and does not imply endorsement by the U.S. Government.

Although this information product, for the most part, is in the public domain, it also may contain copyrighted materials as noted in the text. Permission to reproduce copyrighted items must be secured from the copyright owner.

Suggested citation:

Johnson, T.D., and Belitz, Kenneth, 2014, California Groundwater Units: U.S. Geological Survey Data Series 796, 34 p., http://dx.doi.org/10.3133/ds796.

ISSN 2327-638X (online) 


\section{Contents}

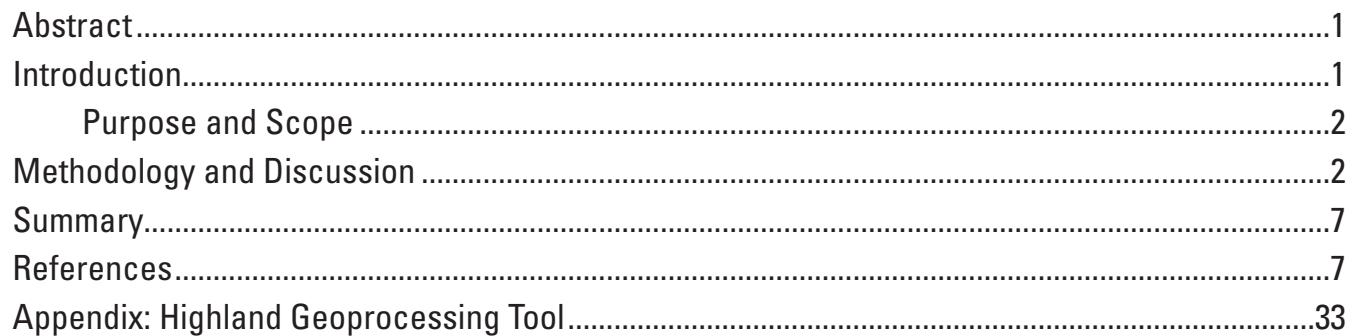

\section{Figures}

1. Map showing California Groundwater Units: southern San Joaquin Valley and southern Sierra Nevada shown ......................................................................................

2. Map showing hydrogeologic provinces of California.......................................................

3. Map showing California Groundwater Units: Statewide map ........................................6

\section{Table}

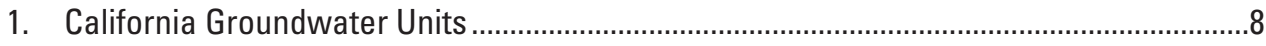

\section{Conversion Factors}

SI to Inch/Pound

\begin{tabular}{lcl}
\hline \multicolumn{1}{c}{ Multiply } & By & \multicolumn{1}{c}{ To obtain } \\
\hline meter (m) & Length & \\
meter (m) & 3.281 & foot (ft) \\
kilometer $(\mathrm{km})$ & 1.094 & yard (yd) \\
kilometer $(\mathrm{km})$ & 0.6214 & mile (mi) \\
& 0.5400 & mile, nautical (nmi) \\
\hline square kilometer $\left(\mathrm{km}^{2}\right)$ & Area & acre \\
square kilometer $\left(\mathrm{km}^{2}\right)$ & 247.1 & square mile $\left(\mathrm{mi}^{2}\right)$ \\
\hline
\end{tabular}




\title{
Abbreviations and Acronyms
}

\author{
DEM Digital Elevation Model \\ CDWR California Department of Water Resources \\ GAMA Groundwater Ambient Monitoring and Assessment Program \\ Gb Groundwater basin \\ GU Groundwater Unit \\ $\mathrm{Hgb} \quad$ Highland area - Groundwater-basin based \\ Hp Highland area - Province-based \\ ID Identification number \\ USGS U.S. Geological Survey
}




\title{
California Groundwater Units
}

\author{
By Tyler D. Johnson and Kenneth Belitz
}

\section{Abstract}

The California Groundwater Units dataset classifies and delineates areas within the State of California into one of three groundwater-based polygon units: (1) those areas previously defined as alluvial groundwater basins or subbasins, (2) highland areas that are adjacent to and topographically upgradient of groundwater basins, and (3) highland areas not associated with a groundwater basin, only a hydrogeologic province. In total, 938 Groundwater Units are represented. The Groundwater Units dataset relates existing groundwater basins with their newly delineated highland areas which can be used in subsequent hydrologic studies. The methods used to delineate groundwater-basin-associated highland areas are similar to those used to delineate a contributing area (such as for a lake or water body); the difference is that highland areas are constrained to the immediately surrounding upslope (upstream) area. Upslope basins have their own delineated highland. A geoprocessing tool was created to facilitate delineation of highland areas for groundwater basins and subbasins and is available for download.

\section{Introduction}

The California Groundwater Units dataset is a digital geospatial representation of areas within the State of California that have been classified and delineated into one of three groundwater-based polygon units: (1) those areas defined as alluvial groundwater basins or subbasins (Gb), initially on the basis of California Department of Water Resources (CDWR) Bulletin 118 (California Department of Water Resources, 2002); (2) highland areas that are adjacent to and topographically upgradient of groundwater basins (Hgb); and (3) highland areas associated with the hydrogeologic province they are within (Hp) instead of a groundwater basin. Four hundred and sixty-three (463) existing groundwater basins (some were termed "subbasins" by the CDWR) were identified, of which 453 have an associated highland area. Ten groundwater basins did not have an associated highland area delineated because the basin was either surrounded by other groundwater basins or adjacent to the ocean. In addition to the 453 highland areas, 22 highland areas not topographically upgradient of groundwater basins previously defined by the CDWR are instead associated with the hydrogeologic province they are located within (as defined in Johnson and Belitz, 2003). In total, 938 Groundwater Units are represented (table 1).

The highland areas that were associated with groundwater basins (453) were delineated on the basis of the surficial contributing area (watershed) immediately adjacent to and upstream of each groundwater basin. The method of delineation is comparable to that used for delineating the contributing area for a lake or water body. Highland areas are not intended to represent the entire surficial contributing area for a groundwater basin, however, because adjacent upslope (upstream) groundwater basins (and their surficial contributing areas) are not included in the delineation of highland areas.

A custom geoprocessing tool, the Highland tool, was created to automate the highland delineation process and is provided for download (appendix). This tool can be used to delineate a highland area if a flow-direction grid (defined in the Methodology and Discussion section) and two or more groundwater basin polygons are provided. The Highland tool is not designed to identify contributing areas; rather, it is designed to identify the local highlands adjacent to and upslope (upstream) of a groundwater basin.

The highland areas that were associated with a hydrogeologic province (22) consisted of those remaining portions of the State that did not have any previously defined groundwater basin located within them, and therefore did not have an associated groundwater-based highland area. These highland areas were assigned the province they were located within and were not delineated by use of any tool. These areas were located at the margins of California that bordered neighboring States, Mexico, or the Pacific Ocean. 


\section{Purpose and Scope}

The Groundwater Unit spatial dataset was developed to classify and delineate all areas of California into three categories for use in subsequent hydrologic studies. Many studies and reports use the CDWR groundwater basin/ subbasin boundaries as the basic study area extent, including the Groundwater Ambient Monitoring and Assessment (GAMA) Program of the California Priority Basin Project (Kulongoski and Belitz, 2004); however, this approach leaves areas of the State outside of groundwater basins unclassified. With the Groundwater Unit dataset, relating existing groundwater basins to their newly delineated highland areas is possible. Highland areas not associated with a groundwater basin were also identified such that all areas of California are now classified into one of three Groundwater Units (463 Gb, $453 \mathrm{Hgb}$, and $22 \mathrm{Hp}$ ).

The purposes of this report are to (1) document and describe the creation of the California Groundwater Unit geospatial dataset and (2) document and describe the Highland tool that was used to generate highland areas. The dataset and the tool are available for download along with this report. Areas outside of California were not considered for this report. Generated highland areas were restricted to the State boundaries, even though a highland could have extended into neighboring States and Mexico if allowed.

\section{Methodology and Discussion}

Identification and delineation of the groundwater units involved three primary steps: (1) identify the predefined alluvial groundwater basins within the State; (2) delineate the associated highland areas of those basins, if any; and (3) assign the remaining highland portions of the State that are not associated with a California groundwater basin to a regional hydrogeologic province.

Step 1 was accomplished by identifying the alluvial groundwater basins established by the CDWR. Because the CDWR basin boundary dataset is periodically updated and because consistency with the GAMA Program is needed, the groundwater basins used by the GAMA Program (Belitz and others, 2003) generally were used for this report. For the GAMA Program, CDWR basin boundaries from 2002 (California Department of Water Resources, 2002) were used to identify groundwater basin units, except in the Central Valley hydrogeologic province, where the subbasin boundaries were used. The Redding area was the sole exception to this rule in the Central Valley hydrogeologic province where the basin boundary was used instead of the subbasin boundary.

Groundwater basins identified in this report deviate from those used by Belitz and others (2003) in a few instances. Volcanic basins, previously defined as a type of groundwater basin, were not included because they have since been redefined by CDWR as "source-areas" (California Department of Water Resources, 2012). Another deviation occurred in the Salinas Valley, which was formerly identified as a single groundwater basin. For the purposes of distinguishing the highland areas of the Paso Robles area from those of the Monterey Bay and Forebay areas, subbasins were grouped to define three groundwater basin units. To reconstruct the Belitz and others (2003) basin designations, these three Groundwater Units may be recombined.

The name and the identification number (ID) for a groundwater basin was taken from either the CDWR basin or subbasin ID. The exception is in the Salinas Valley, where the groundwater subbasins were grouped. In this area, the Groundwater Units are identified as 3-4.1 and 3-4.2, with Paso Robles being its own preexisting stand-alone subbasin (3-4.06). (The CDWR basin ID for Salinas Valley is 3-4.) The names and IDs of the subbasins that were grouped to form 3-4.1 and 3-4.2 are listed in table 1.

Step 2 was accomplished by developing a custom tool (hereinafter referred to as the Highland geoprocessing tool, described in the appendix) in Esri's ArcGIS Model Builder software (Esri, 2011a) to automate the highland delineation process. The tool invokes a number of processes, but primarily uses the Watershed tool (Esri, 2011b) to create a highland polygon for any given groundwater basin using the basin boundary dataset described in step 1 and a flow-direction grid (described below) as inputs. The Highland tool delineates one highland area for each groundwater basin, as appropriate. At least two basins must be included in the input because the tool is designed to delineate a highland area with one or more upgradient (upslope) basins. The Watershed tool is sufficient for delineating a highland with no upgradient (upslope) basins. The Highland tool iterates through each basin one at a time, delineating the highland areas. Once highland areas were created for the appropriate basins, they were merged together into one geodataset in a separate process outside of the Highland tool.

One of the two datasets required for the Highland tool is a flow-direction grid. A flow-direction grid is a pixel-based dataset that identifies which direction a hypothetical drop of water would flow in each cell within a gridded extent. Every cell within the grid will therefore have a direction of flow assigned to it. The flow-direction grid is derived from 
a digital elevation model (DEM). Therefore, a DEM must be sufficiently large to capture the entire extent. DEMs were downloaded from the National Hydrography Dataset Plus website (http://www.horizon-systems.com/nhdplus/index. pHp). Three DEMs were merged together (also known as "mosaicing") to attain a single DEM that covered the entire State. Due to the large size of California, and consequently very large file size and processing times, the DEM was downscaled (using bilinear interpolation) (Esri, 2011c) from 30-meter (m) to 300-m grid cells.

The scale (pixel size) used to develop the dataset was partly determined by the size of the CDWR groundwater basin units. The smallest CDWR groundwater basin was less than 1.4 square kilometers $\left(\mathrm{km}^{2}\right)$, therefore, a scale smaller than this was needed to properly delineate a contributing highland. Scale was also limited by the processing time and computational resources available to calculate a dataset on a statewide extent. Therefore, a 300-m pixel size was chosen as an acceptable balance. The smallest highland area delineated was less than $0.5 \mathrm{~km}^{2}$. Choosing an appropriate pixel size is important when delineating highland areas to avoid incomplete highland areas when the pixel size is too small or overly generalized highland areas when the pixel size is too large.

One downside of scaling a DEM to a larger cell size is that flat or low-lying areas, such as stream channels or lakes, can become generalized, resulting in situations where water no longer flows through the digital representation of the flow system, or flows in incorrect directions. In order to retain hydrologic continuity, a regional stream dataset (polyline) and a lakes dataset (polygon) (both acquired from Esri) were used to "burn" the hydrologic flow back into the DEM. This burning process effectively forces the flow to follow the stream channels and proceed through lakes by lowering the cell elevations in these areas by a specified amount. The cell values for lakes and streams were lowered by $20 \mathrm{~m}$ using a conditional expression with the Raster Calculator tool (Esri, 2011d). Lakes smaller than $1 \mathrm{~km}^{2}$ were excluded, as well as all canals, ditches, and ice masses. Finally, any remaining "sinks," or imperfections in the DEM that prevent flow out of the cell were "filled" by raising their elevation to the surrounding cell values using the "Fill" tool (Esri, 2011e). The Fill tool was allowed to fill sinks unrestrained. Once a hydrologically consistent DEM is built, a flow-direction grid can be created using the Flow Direction tool (Esri, 2011f).

Many groundwater basins in the Central Valley hydrogeologic province are separated by a river: one groundwater basin along the right bank, and another along the left bank. Therefore, each groundwater basin has its own highland area delineated for each side of the river, which can be desirable, especially when the geologic content of the two highland areas are different. An example of this is in the Central Valley hydrogeologic province where the San Joaquin and Sacramento Rivers bisect groundwater basins into left- and right-bank basins. The geology in the Sierra Nevada hydrogeologic province differs from the geology of the Northern and Southern Coast Ranges hydrogeologic provinces; therefore, dividing basins into western and eastern portions may be beneficial in identifying correlations between source rocks and water quality. Conversely, it may not be desirable to subdivide basins where the surrounding rocks are similar. In these cases, highland areas and basins can be grouped to create more generalized Groundwater Units.

Due to the nature of the pixel-based methodology, a river and all of its contributing upslope highland area could be focused into a single downstream pixel where it encounters two groundwater basins on either side of the river (pixel). However, this pixel can be assigned to only one groundwater basin. As a result, one groundwater basin could possibly receive all of the upslope contributing highland area rather than both basins receiving it. In these cases, it may be beneficial to combine the groundwater basins and, likewise, the corresponding highland areas.

By using the flow-direction grid and the 463 CDWR basin boundaries, 453 highland areas were delineated by using the Highland tool. The identifying number for a highland area is derived from using the corresponding groundwater basin ID and adding "Hgb-" for "Highland area - Groundwater Basin based" as a prefix. Similarly, the name for a highland area is derived by adding "Highlands" as a suffix to the name of the corresponding groundwater basin. For example, groundwater basin "6-12, Owens Valley" will have a corresponding highland area named "Hgb-6-12, Owens Valley Highlands."

A number of groundwater basins and their highland areas are shown in figure 1. Notice that the delineated highland area for groundwater basin 5-22.14 does not include the highland area delineated for groundwater basins 5-25, 5-26, 5-27, or 5-28. In a normal watershed delineation procedure, all of these groundwater basins and highlands would be included in the highland delineation for 5-22.14 because they all flow into it. Similarly, 5-22.14 would flow into both 5-22.12 and 5-22.13, and so on, up the San Joaquin Valley. Instead, the highland area for 5-22.14 contains only its immediate contributing area, stopping at any adjacent Groundwater Unit. Groundwater basin 5-22.12 does not have a highland area delineated because it is surrounded by other groundwater basins. 


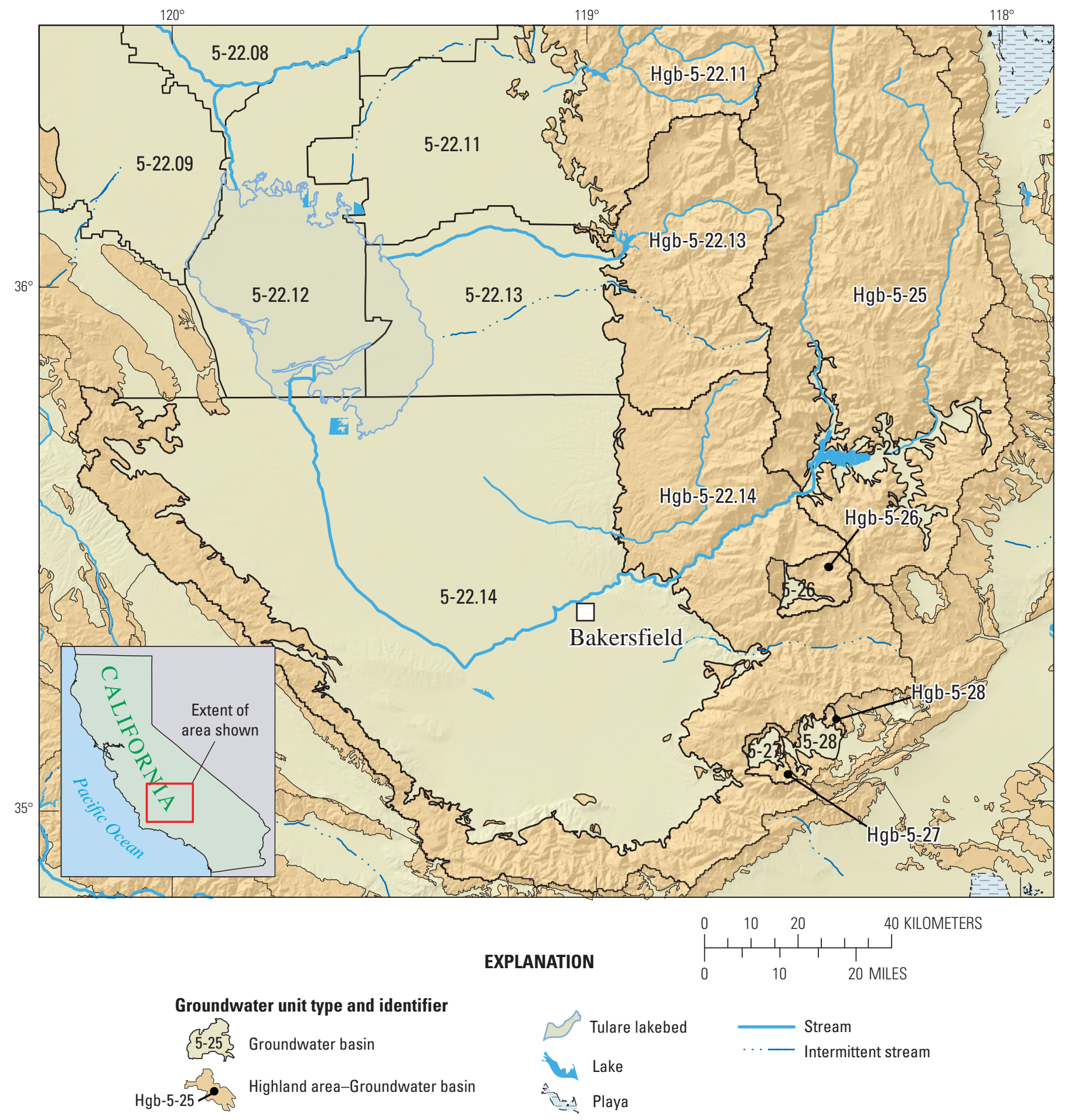

Figure 1. California Groundwater Units: southern San Joaquin Valley and southern Sierra Nevada shown. (For figure clarity, not all groundwater basins and highlands are labeled.) 
Step 3 involved identifying and classifying the remaining areas of the State that were not part of either a groundwater basin or a groundwater basin highland. These areas were identified by using the California State boundary (California Department of Fish and Game, 2004) to enclose any areas that were not identified as belonging to a California groundwater basin or groundwater basin highland. These areas were at the coastline and at the borders of adjacent States and Mexico. The polygons were then assigned and named by using the local hydrogeologic province and a local feature. The Groundwater Unit identification numbers for these areas are derived from the names of the 10 hydrogeologic provinces of California (fig. 2) and preceded with an "Hp" to indicate "Highland area - Province based." Numbering is sequential from north to south, with the first number indicating the province and the number after the decimal place indicating the subarea. For example, Hp-1.4 is in the first province (Northern Coast Ranges) and is the fourth subarea (Point Reyes).

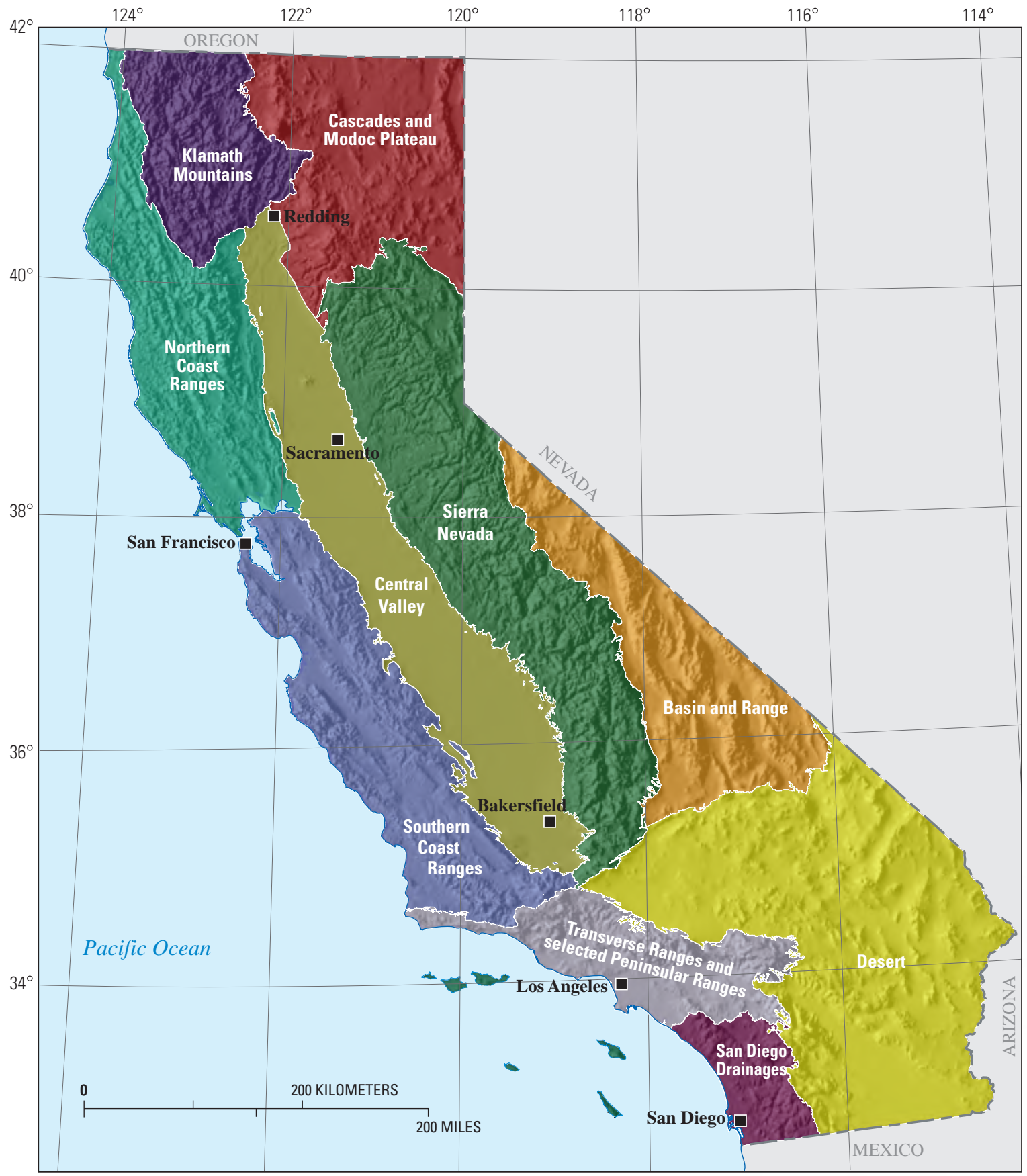

Shaded relief derived from U.S. Geological Survey

Provinces from Belitz and others, 2003 National Elevation Dataset, 2006

Albers Equal Area Conic Projection

Figure 2. Hydrogeologic provinces of California. 
After merging the groundwater basins and highland areas into one geodataset, small sliver "artifact” polygons resulted. These occurred mostly because the highland areas, which were derived from a 300-m-pixel grid, did not match up with the detailed coastline of California, which is a polyline dataset.
These artifacts were removed wherever possible. Figure 3 shows the three types of Groundwater Units delineated for the entire State. A digital geospatial representation of the Groundwater Units, which can be viewed in a GIS software package, is available for download at http://pubs.usgs.gov/ ds/796/.

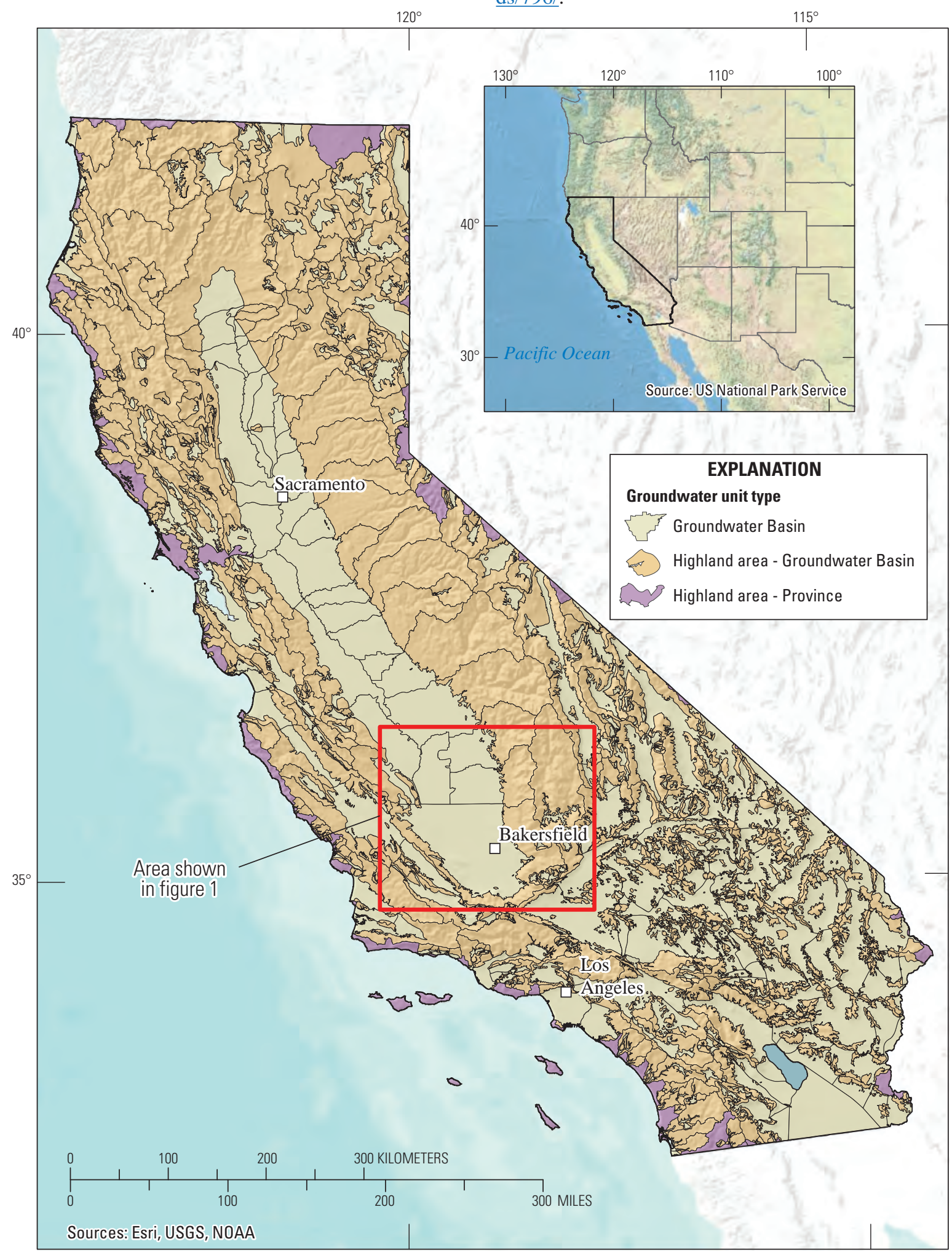

Figure 3. California Groundwater Units: Statewide map. 


\section{Summary}

Three types of Groundwater Units have been defined for the State of California: (1) alluvial groundwater basins, (2) highland areas associated with a groundwater basin, and (3) highland areas not associated with a groundwater basin. The established CDWR groundwater basin and subbasin boundaries formed the origin of the groundwater basin units. The highland areas were delineated by identifying topographically upgradient areas for each groundwater basin. To facilitate this process, a modified DEM and flowdirection grid were created for the State, and a custom tool was built to automate the delineation process. Areas along the edges of the State that did not belong to a groundwater basin or a delineated highland were classified as a provincebased highland area. The Groundwater Units dataset and the Highland tool are available for download along with this report at http://pubs.usgs.gov/ds/796/.

\section{References}

Belitz, Kenneth, Dubrovsky, N.M., Burow, K., Jurgens, B., and Johnson, T., 2003, Framework for a ground-water quality monitoring and assessment program for California: U.S. Geological Survey Water-Resources Investigations Report 03-4166, 78 p. (Also available at http://pubs.usgs. gov/wri/wri034166/.)

California Department of Fish and Game, 2004, Digital geospatial data of the counties of California (cnty24k97_1). Dataset is no longer available and has been superseded and updated by California Department of Forestry and Fire Protection, 2009, Cnty24k09_1, accessed March 21, 2014, at http://www.atlas.ca.gov/download.html\#/casil/ boundaries.

California Department of Water Resources, 2002, Groundwater Basin Map Draft Version 2 (May 3, 2002, no longer available), Bulletin 118, 246 p.
California Department of Water Resources, 2012, Groundwater Basin Map Version 4.1 (June 20, 2012), Bulletin 118, accessed March 7, 2014, at http://www. water.ca.gov/groundwater/bulletin118/gwbasin maps descriptions.cfm.

Esri, 2011a, ArcGIS Resource Center-What is ModelBuilder?, accessed May 2012 at http://help. arcgis.com/en/arcgisdesktop/10.0/help/index. html\#//002w00000001000000.

Esri, 2011b, ArcGIS Resource Center-Watershed (Spatial Analyst), accessed May 2012 at http:// help.arcgis.com/en/arcgisdesktop/10.0/help/index. html\#//009z00000059000000.htm.

Esri, 2011c, ArcGIS Resource Center-Resample (Data Management), accessed May 2012 at http:// help.arcgis.com/en/arcgisdesktop/10.0/help/index. html\#//00170000009t000000.

Esri, 2011d, ArcGIS Resource Center-Raster Calculator (Spatial Analyst), accessed May 2012 at http:// help.arcgis.com/en/arcgisdesktop/10.0/help/index. html\#//009z000000z7000000.htm.

Esri, 2011e, ArcGIS Resource Center-Fill (Spatial Analyst), accessed May 2012 at http://help. arcgis.com/en/arcgisdesktop/10.0/help/index. html\#//009z00000050000000.htm.

Esri, 2011f, ArcGIS Resource Center-Flow Direction (Spatial Analyst), accessed May 2012 at http:// help.arcgis.com/en/arcgisdesktop/10.0/help/index. html\#//009z00000052000000.htm.

Johnson, Tyler, and Belitz, Kenneth, 2003, Hydrogeologic provinces for California based upon established groundwater basins and watershed polygons, accessed March 7, 2014, at http://water.usgs.gov/GIS/metadata/ usgswrd/XML/ca provinces.Xml.

Kulongoski, J.T., and Belitz, Kenneth, 2004, Ground-Water Ambient Monitoring and Assessment Program: U.S. Geological Survey Fact Sheet 2004-3088, 2 p. (Also available at http://pubs.usgs.gov/fs/2004/3088/.) 
Table 1. California Groundwater Units.

[GU ID is the unique groundwater unit identifier. GU name is the groundwater unit name. GU names in uppercase are the same names as those used in CDWR 2002 groundwater basin map publication except for the word "HIGHLANDS" when appended. GU type identifies one of three types of groundwater units: Groundwater basin, Highland area - Gb (groundwater-basin-based highland), or Highland area - Province (province-based highland). Related Gb references the basin-ID or subbasin-ID in the CDWR 2002 groundwater basin map publication. CDWR, California Department of Water Resources; km², square kilometer; —, no data]

\begin{tabular}{|c|c|c|c|c|}
\hline $\begin{array}{l}\text { GU } \\
\text { ID }\end{array}$ & $\begin{array}{c}\text { GU } \\
\text { Name }\end{array}$ & $\begin{array}{l}\text { GU } \\
\text { Type }\end{array}$ & $\begin{array}{l}\text { Area } \\
\left(\mathbf{k m}^{2}\right)\end{array}$ & $\begin{array}{c}\text { Related } \\
\text { Gb }\end{array}$ \\
\hline $1-1$ & SMITH RIVER PLAIN & Groundwater Basin & 163.8 & $1-1$ \\
\hline $1-10$ & EEL RIVER VALLEY & Groundwater Basin & 298.4 & $1-10$ \\
\hline $1-11$ & COVELO ROUND VALLEY & Groundwater Basin & 66.4 & $1-11$ \\
\hline $1-12$ & LAYTONVILLE VALLEY & Groundwater Basin & 20.3 & $1-12$ \\
\hline $1-13$ & LITTLE LAKE VALLEY & Groundwater Basin & 40.6 & $1-13$ \\
\hline $1-14$ & LOWER KLAMATH RIVER VALLEY & Groundwater Basin & 28.4 & $1-14$ \\
\hline $1-15$ & HAPPY CAMP TOWN AREA & Groundwater Basin & 11.2 & $1-15$ \\
\hline $1-16$ & SEIAD VALLEY & Groundwater Basin & 9.1 & $1-16$ \\
\hline $1-17$ & BRAY TOWN AREA & Groundwater Basin & 32.5 & $1-17$ \\
\hline $1-18$ & RED ROCK VALLEY & Groundwater Basin & 36.4 & $1-18$ \\
\hline $1-19$ & ANDERSON VALLEY & Groundwater Basin & 20.1 & $1-19$ \\
\hline $1-2$ & KLAMATH RIVER VALLEY & Groundwater Basin & 652.9 & $1-2$ \\
\hline $1-20$ & GARCIA RIVER VALLEY & Groundwater Basin & 9.1 & $1-20$ \\
\hline $1-21$ & FORT BRAGG TERRACE AREA & Groundwater Basin & 97.5 & $1-21$ \\
\hline $1-22$ & FAIRCHILD SWAMP VALLEY & Groundwater Basin & 13.3 & $1-22$ \\
\hline $1-25$ & PRAIRIE CREEK AREA & Groundwater Basin & 81.0 & $1-25$ \\
\hline $1-26$ & REDWOOD CREEK AREA & Groundwater Basin & 8.1 & $1-26$ \\
\hline $1-27$ & BIG LAGOON AREA & Groundwater Basin & 54.0 & $1-27$ \\
\hline $1-28$ & MATTOLE RIVER VALLEY & Groundwater Basin & 12.8 & $1-28$ \\
\hline $1-29$ & HONEYDEW TOWN AREA & Groundwater Basin & 9.6 & $1-29$ \\
\hline $1-3$ & BUTTE VALLEY & Groundwater Basin & 322.7 & $1-3$ \\
\hline $1-30$ & PEPPERWOOD TOWN AREA & Groundwater Basin & 25.5 & $1-30$ \\
\hline $1-31$ & WEOTT TOWN AREA & Groundwater Basin & 14.8 & $1-31$ \\
\hline $1-32$ & GARBERVILLE TOWN AREA & Groundwater Basin & 8.6 & $1-32$ \\
\hline $1-33$ & LARABEE VALLEY & Groundwater Basin & 3.9 & $1-33$ \\
\hline $1-34$ & DINSMORES TOWN AREA & Groundwater Basin & 9.2 & $1-34$ \\
\hline $1-35$ & HYAMPOM VALLEY & Groundwater Basin & 5.5 & $1-35$ \\
\hline $1-36$ & HETTENSHAW VALLEY & Groundwater Basin & 3.4 & $1-36$ \\
\hline $1-37$ & COTTONEVA CREEK VALLEY & Groundwater Basin & 3.1 & $1-37$ \\
\hline $1-38$ & LOWER LAYTONVILLE VALLEY & Groundwater Basin & 8.7 & $1-38$ \\
\hline $1-39$ & BRANSCOMB TOWN AREA & Groundwater Basin & 5.6 & $1-39$ \\
\hline $1-4$ & SHASTA VALLEY & Groundwater Basin & 792.9 & $1-4$ \\
\hline $1-40$ & TEN MILE RIVER VALLEY & Groundwater Basin & 6.0 & $1-40$ \\
\hline $1-41$ & LITTLE VALLEY & Groundwater Basin & 3.3 & $1-41$ \\
\hline $1-42$ & SHERWOOD VALLEY & Groundwater Basin & 4.7 & $1-42$ \\
\hline $1-43$ & WILLIAMS VALLEY & Groundwater Basin & 6.7 & $1-43$ \\
\hline $1-44$ & EDEN VALLEY & Groundwater Basin & 5.6 & $1-44$ \\
\hline $1-45$ & BIG RIVER VALLEY & Groundwater Basin & 6.8 & $1-45$ \\
\hline
\end{tabular}


Table 1. California Groundwater Units.-Continued

[GU ID is the unique groundwater unit identifier. GU name is the groundwater unit name. GU names in uppercase are the same names as those used in CDWR 2002 groundwater basin map publication except for the word "HIGHLANDS" when appended. GU type identifies one of three types of groundwater units: Groundwater basin, Highland area - Gb (groundwater-basin-based highland), or Highland area - Province (province-based highland). Related Gb references the basin-ID or subbasin-ID in the CDWR 2002 groundwater basin map publication. CDWR, California Department of Water Resources; km², square kilometer; -, no data]

\begin{tabular}{|c|c|c|c|c|}
\hline $\begin{array}{l}\text { GU } \\
\text { ID }\end{array}$ & $\begin{array}{c}\text { GU } \\
\text { Name }\end{array}$ & $\begin{array}{l}\text { GU } \\
\text { Type }\end{array}$ & $\begin{array}{l}\text { Area } \\
\left(\mathbf{k m}^{2}\right)\end{array}$ & $\begin{array}{c}\text { Related } \\
\text { Gb }\end{array}$ \\
\hline $1-46$ & NAVARRO RIVER VALLEY & Groundwater Basin & 3.1 & $1-46$ \\
\hline $1-48$ & GRAVELLY VALLEY & Groundwater Basin & 12.0 & $1-48$ \\
\hline $1-49$ & ANNAPOLIS OHLSON RANCH FM HIGHLANDS & Groundwater Basin & 35.0 & $1-49$ \\
\hline $1-5$ & SCOTT RIVER VALLEY & Groundwater Basin & 258.3 & $1-5$ \\
\hline $1-50$ & KNIGHTS VALLEY & Groundwater Basin & 16.5 & $1-50$ \\
\hline $1-51$ & POTTER VALLEY & Groundwater Basin & 33.4 & $1-51$ \\
\hline $1-52$ & UKIAH VALLEY & Groundwater Basin & 151.9 & $1-52$ \\
\hline $1-53$ & SANEL VALLEY & Groundwater Basin & 22.6 & $1-53$ \\
\hline $1-54$ & ALEXANDER VALLEY & Groundwater Basin & 125.5 & $1-54$ \\
\hline $1-55$ & SANTA ROSA VALLEY & Groundwater Basin & 409.1 & $1-55$ \\
\hline $1-56$ & McDOWELL VALLEY & Groundwater Basin & 6.0 & $1-56$ \\
\hline $1-57$ & BODEGA BAY AREA & Groundwater Basin & 10.8 & $1-57$ \\
\hline $1-59$ & WILSON GROVE FORMATION HIGHLANDS & Groundwater Basin & 349.9 & $1-59$ \\
\hline $1-6$ & HAYFORK VALLEY & Groundwater Basin & 13.3 & $1-6$ \\
\hline $1-60$ & LOWER RUSSIAN RIVER VALLEY & Groundwater Basin & 26.9 & $1-60$ \\
\hline $1-61$ & FORT ROSS TERRACE DEPOSITS & Groundwater Basin & 34.4 & $1-61$ \\
\hline $1-62$ & WILSON POINT AREA & Groundwater Basin & 2.9 & $1-62$ \\
\hline $1-7$ & HOOPA VALLEY & Groundwater Basin & 15.8 & $1-7$ \\
\hline $1-8$ & MAD RIVER VALLEY & Groundwater Basin & 160.2 & $1-8$ \\
\hline $1-9$ & EUREKA PLAIN & Groundwater Basin & 151.5 & $1-9$ \\
\hline $2-1$ & PETALUMA VALLEY & Groundwater Basin & 186.5 & $2-1$ \\
\hline $2-10$ & LIVERMORE VALLEY & Groundwater Basin & 281.5 & $2-10$ \\
\hline $2-11$ & SUNOL VALLEY & Groundwater Basin & 67.3 & $2-11$ \\
\hline $2-19$ & KENWOOD VALLEY & Groundwater Basin & 20.8 & $2-19$ \\
\hline $2-2$ & NAPA-SONOMA VALLEY & Groundwater Basin & 530.4 & $2-2$ \\
\hline $2-22$ & HALF MOON BAY TERRACE & Groundwater Basin & 37.2 & $2-22$ \\
\hline $2-24$ & SAN GREGORIO VALLEY & Groundwater Basin & 4.3 & $2-24$ \\
\hline $2-26$ & PESCADERO VALLEY & Groundwater Basin & 11.8 & $2-26$ \\
\hline $2-27$ & SAND POINT AREA & Groundwater Basin & 5.7 & $2-27$ \\
\hline $2-28$ & ROSS VALLEY & Groundwater Basin & 7.1 & $2-28$ \\
\hline $2-29$ & SAN RAFAEL VALLEY & Groundwater Basin & 3.5 & $2-29$ \\
\hline $2-3$ & SUISUN-FAIRFIELD VALLEY & Groundwater Basin & 540.6 & $2-3$ \\
\hline $2-30$ & NOVATO VALLEY & Groundwater Basin & 83.1 & $2-30$ \\
\hline $2-31$ & ARROYO DEL HAMBRE VALLEY & Groundwater Basin & 3.2 & $2-31$ \\
\hline $2-32$ & VISITACION VALLEY & Groundwater Basin & 23.6 & $2-32$ \\
\hline $2-33$ & ISLAIS VALLEY & Groundwater Basin & 24.0 & $2-33$ \\
\hline $2-35$ & WESTSIDE & Groundwater Basin & 102.8 & $2-35$ \\
\hline $2-36$ & SAN PEDRO VALLEY & Groundwater Basin & 2.8 & $2-36$ \\
\hline
\end{tabular}


Table 1. California Groundwater Units.-Continued

[GU ID is the unique groundwater unit identifier. GU name is the groundwater unit name. GU names in uppercase are the same names as those used in CDWR 2002 groundwater basin map publication except for the word "HIGHLANDS" when appended. GU type identifies one of three types of groundwater units: Groundwater basin, Highland area - Gb (groundwater-basin-based highland), or Highland area - Province (province-based highland). Related Gb references the basin-ID or subbasin-ID in the CDWR 2002 groundwater basin map publication. CDWR, California Department of Water Resources; km², square kilometer; —, no data]

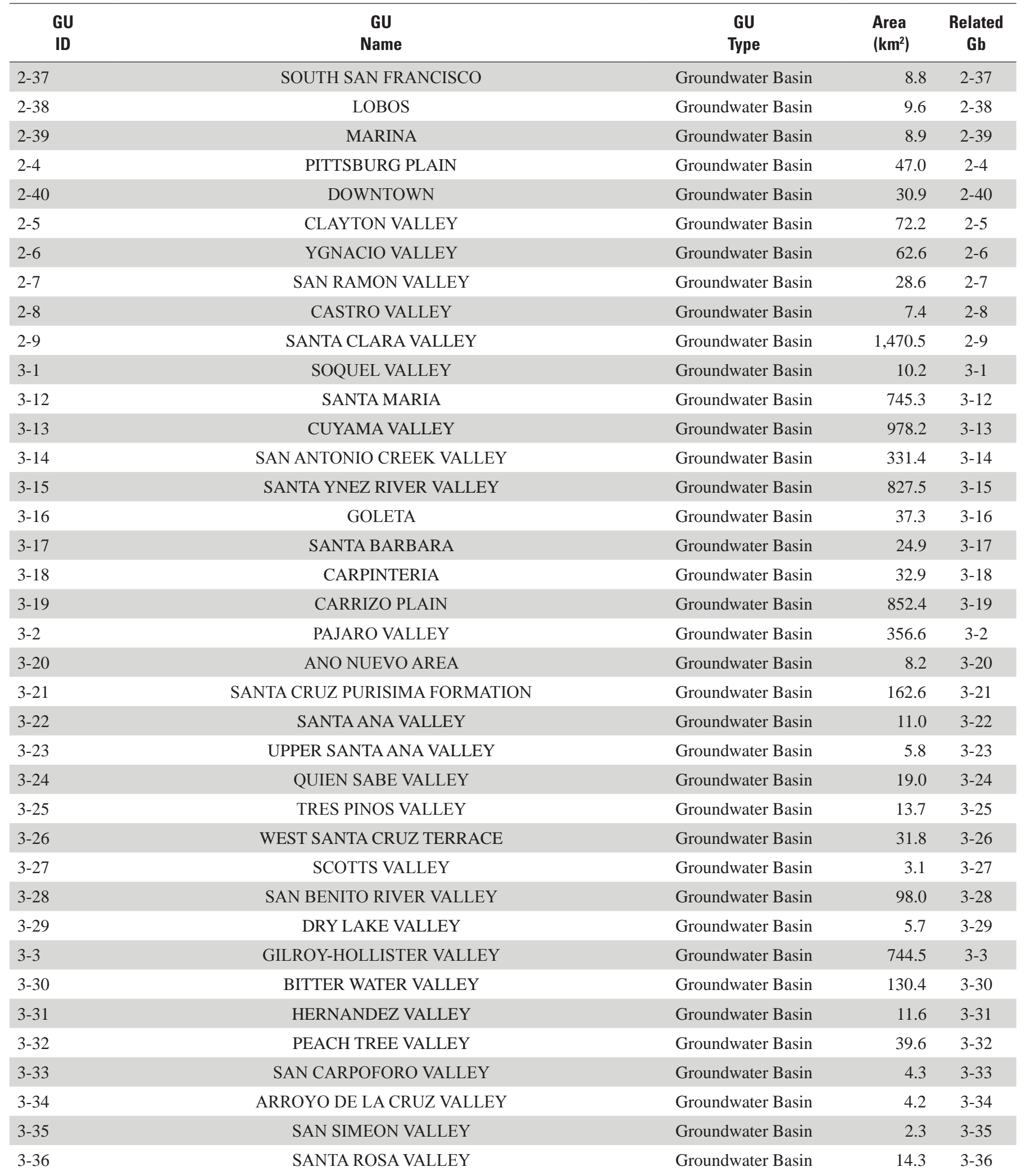


Table 1. California Groundwater Units.-Continued

[GU ID is the unique groundwater unit identifier. GU name is the groundwater unit name. GU names in uppercase are the same names as those used in CDWR 2002 groundwater basin map publication except for the word "HIGHLANDS" when appended. GU type identifies one of three types of groundwater units: Groundwater basin, Highland area - Gb (groundwater-basin-based highland), or Highland area - Province (province-based highland). Related Gb references the basin-ID or subbasin-ID in the CDWR 2002 groundwater basin map publication. CDWR, California Department of Water Resources; km², square kilometer; —, no data]

\begin{tabular}{|c|c|c|c|c|}
\hline $\begin{array}{l}\text { GU } \\
\text { ID }\end{array}$ & $\begin{array}{l}\text { GU } \\
\text { Name }\end{array}$ & $\begin{array}{l}\text { GU } \\
\text { Type }\end{array}$ & $\begin{array}{l}\text { Area } \\
\left(\mathbf{k m}^{2}\right)\end{array}$ & $\begin{array}{l}\text { Related } \\
\text { Gb }\end{array}$ \\
\hline 3-37 & VILLA VALLEY & Groundwater Basin & 5.5 & $3-37$ \\
\hline 3-38 & CAYUCOS VALLEY & Groundwater Basin & 1.4 & 3-38 \\
\hline 3-39 & OLD VALLEY & Groundwater Basin & 4.8 & 3-39 \\
\hline $3-4.1$ & $\begin{array}{l}\text { 180/400 FOOT AQUIFER; EAST SIDE AQUIFER; SEASIDE AREA; } \\
\text { LANGLEY AREA; CORRAL DE TIERRA AREA }\end{array}$ & Groundwater Basin & 831.2 & $\begin{array}{l}3-4.01 ; \\
3-4.02 \\
3-4.08 \\
3-4.09 \\
3-4.10\end{array}$ \\
\hline $3-4.2$ & FOREBAY AQUIFER; UPPER VALLEY AQUIFER & Groundwater Basin & 778.0 & $\begin{array}{r}3-4.04 \\
3-4.05\end{array}$ \\
\hline 3-4.06 & PASO ROBLES AREA & Groundwater Basin & $2,415.7$ & 3-4.06 \\
\hline $3-40$ & TORO VALLEY & Groundwater Basin & 2.9 & $3-40$ \\
\hline 3-41 & MORRO VALLEY & Groundwater Basin & 2.6 & $3-41$ \\
\hline 3-42 & CHORRO VALLEY & Groundwater Basin & 6.3 & $3-42$ \\
\hline 3-43 & RINCONADA VALLEY & Groundwater Basin & 10.4 & $3-43$ \\
\hline 3-44 & POZO VALLEY & Groundwater Basin & 27.7 & $3-44$ \\
\hline 3-45 & HUASNA VALLEY & Groundwater Basin & 19.0 & $3-45$ \\
\hline $3-46$ & RAFAEL VALLEY & Groundwater Basin & 12.1 & $3-46$ \\
\hline $3-47$ & BIG SPRING AREA & Groundwater Basin & 29.6 & $3-47$ \\
\hline $3-49$ & MONTECITO & Groundwater Basin & 25.4 & $3-49$ \\
\hline 3-5 & CHOLAME VALLEY & Groundwater Basin & 161.2 & $3-5$ \\
\hline 3-50 & FELTON AREA & Groundwater Basin & 4.7 & $3-50$ \\
\hline 3-51 & MAJORS CREEK & Groundwater Basin & 1.5 & $3-51$ \\
\hline 3-52 & NEEDLE ROCK POINT & Groundwater Basin & 1.9 & $3-52$ \\
\hline 3-53 & FOOTHILL & Groundwater Basin & 12.6 & $3-53$ \\
\hline $3-6$ & LOCKWOOD VALLEY & Groundwater Basin & 242.6 & 3-6 \\
\hline 3-7 & CARMEL VALLEY & Groundwater Basin & 20.9 & 3-7 \\
\hline 3-8 & LOS OSOS VALLEY & Groundwater Basin & 28.3 & 3-8 \\
\hline 3-9 & SAN LUIS OBISPO VALLEY & Groundwater Basin & 51.5 & 3-9 \\
\hline $4-1$ & UPPER OJAI VALLEY & Groundwater Basin & 15.4 & $4-1$ \\
\hline $4-10$ & CONEJO & Groundwater Basin & 76.1 & $4-10$ \\
\hline $4-11$ & COASTAL PLAIN OF LOS ANGELES & Groundwater Basin & $1,274.2$ & $4-11$ \\
\hline $4-12$ & SAN FERNANDO VALLEY & Groundwater Basin & 586.1 & $4-12$ \\
\hline $4-13$ & SAN GABRIEL VALLEY & Groundwater Basin & 512.7 & $4-13$ \\
\hline $4-15$ & TIERRA REJADA & Groundwater Basin & 18.6 & $4-15$ \\
\hline $4-16$ & HIDDEN VALLEY & Groundwater Basin & 8.9 & $4-16$ \\
\hline $4-17$ & LOCKWOOD VALLEY & Groundwater Basin & 88.2 & $4-17$ \\
\hline $4-18$ & HUNGRY VALLEY & Groundwater Basin & 21.5 & $4-18$ \\
\hline $4-19$ & THOUSAND OAKS AREA & Groundwater Basin & 12.6 & $4-19$ \\
\hline
\end{tabular}


Table 1. California Groundwater Units.—Continued

[GU ID is the unique groundwater unit identifier. GU name is the groundwater unit name. GU names in uppercase are the same names as those used in CDWR 2002 groundwater basin map publication except for the word "HIGHLANDS" when appended. GU type identifies one of three types of groundwater units: Groundwater basin, Highland area - Gb (groundwater-basin-based highland), or Highland area - Province (province-based highland). Related Gb references the basin-ID or subbasin-ID in the CDWR 2002 groundwater basin map publication. CDWR, California Department of Water Resources; km², square kilometer; —, no data]

\begin{tabular}{|c|c|c|c|c|}
\hline $\begin{array}{l}\text { GU } \\
\text { ID }\end{array}$ & $\begin{array}{c}\text { GU } \\
\text { Name }\end{array}$ & $\begin{array}{l}\text { GU } \\
\text { Type }\end{array}$ & $\begin{array}{l}\text { Area } \\
\left(\mathbf{k m}^{2}\right)\end{array}$ & $\begin{array}{c}\text { Related } \\
\text { Gb }\end{array}$ \\
\hline $4-2$ & OJAI VALLEY & Groundwater Basin & 27.7 & $4-2$ \\
\hline $4-20$ & RUSSELL VALLEY & Groundwater Basin & 12.5 & $4-20$ \\
\hline $4-22$ & MALIBU VALLEY & Groundwater Basin & 2.5 & $4-22$ \\
\hline $4-23$ & RAYMOND & Groundwater Basin & 106.0 & $4-23$ \\
\hline $4-3$ & VENTURA RIVER VALLEY & Groundwater Basin & 51.5 & $4-3$ \\
\hline $4-4$ & SANTA CLARA RIVER VALLEY & Groundwater Basin & 775.4 & $4-4$ \\
\hline $4-5$ & ACTON VALLEY & Groundwater Basin & 33.5 & $4-5$ \\
\hline $4-6$ & PLEASANT VALLEY & Groundwater Basin & 87.4 & $4-6$ \\
\hline $4-7$ & ARROYO SANTA ROSA VALLEY & Groundwater Basin & 15.1 & $4-7$ \\
\hline $4-8$ & LAS POSAS VALLEY & Groundwater Basin & 170.9 & $4-8$ \\
\hline $4-9$ & SIMI VALLEY & Groundwater Basin & 49.2 & $4-9$ \\
\hline $5-1$ & GOOSE LAKE & Groundwater Basin & 220.1 & $5-1$ \\
\hline $5-10$ & AMERICAN VALLEY & Groundwater Basin & 27.5 & $5-10$ \\
\hline $5-11$ & MOHAWK VALLEY & Groundwater Basin & 76.8 & $5-11$ \\
\hline $5-12$ & SIERRA VALLEY & Groundwater Basin & 514.7 & $5-12$ \\
\hline $5-13$ & UPPER LAKE VALLEY & Groundwater Basin & 29.4 & $5-13$ \\
\hline $5-14$ & SCOTTS VALLEY & Groundwater Basin & 29.6 & $5-14$ \\
\hline $5-15$ & BIG VALLEY & Groundwater Basin & 98.1 & $5-15$ \\
\hline $5-16$ & HIGH VALLEY & Groundwater Basin & 9.5 & $5-16$ \\
\hline $5-17$ & BURNS VALLEY & Groundwater Basin & 11.6 & $5-17$ \\
\hline $5-18$ & COYOTE VALLEY & Groundwater Basin & 26.4 & $5-18$ \\
\hline $5-19$ & COLLAYOMI VALLEY & Groundwater Basin & 26.3 & $5-19$ \\
\hline $5-2$ & ALTURAS AREA & Groundwater Basin & 737.1 & $5-2$ \\
\hline $5-20$ & BERRYESSA VALLEY & Groundwater Basin & 5.6 & $5-20$ \\
\hline $5-21.50$ & RED BLUFF & Groundwater Basin & $1,079.3$ & $5-21.50$ \\
\hline $5-21.51$ & CORNING & Groundwater Basin & 832.1 & $5-21.51$ \\
\hline $5-21.52$ & COLUSA & Groundwater Basin & $3,716.6$ & $5-21.52$ \\
\hline $5-21.53$ & BEND & Groundwater Basin & 84.0 & $5-21.53$ \\
\hline $5-21.54$ & ANTELOPE & Groundwater Basin & 75.7 & $5-21.54$ \\
\hline $5-21.55$ & DYE CREEK & Groundwater Basin & 112.2 & $5-21.55$ \\
\hline $5-21.56$ & LOS MOLINOS & Groundwater Basin & 134.2 & $5-21.56$ \\
\hline $5-21.57$ & VINA & Groundwater Basin & 504.5 & $5-21.57$ \\
\hline $5-21.58$ & WEST BUTTE & Groundwater Basin & 734.8 & $5-21.58$ \\
\hline $5-21.59$ & EAST BUTTE & Groundwater Basin & $1,074.2$ & $5-21.59$ \\
\hline $5-21.60$ & NORTH YUBA & Groundwater Basin & 417.6 & $5-21.60$ \\
\hline $5-21.61$ & SOUTH YUBA & Groundwater Basin & 423.0 & $5-21.61$ \\
\hline $5-21.62$ & SUTTER & Groundwater Basin & 531.6 & $5-21.62$ \\
\hline $5-21.63$ & WEST SUTTER & Groundwater Basin & 416.9 & $5-21.63$ \\
\hline
\end{tabular}


Table 1. California Groundwater Units.-Continued

[GU ID is the unique groundwater unit identifier. GU name is the groundwater unit name. GU names in uppercase are the same names as those used in CDWR 2002 groundwater basin map publication except for the word "HIGHLANDS" when appended. GU type identifies one of three types of groundwater units: Groundwater basin, Highland area - Gb (groundwater-basin-based highland), or Highland area - Province (province-based highland). Related Gb references the basin-ID or subbasin-ID in the CDWR 2002 groundwater basin map publication. CDWR, California Department of Water Resources; km², square kilometer; —, no data]

\begin{tabular}{|c|c|c|c|c|}
\hline $\begin{array}{l}\text { GU } \\
\text { ID }\end{array}$ & $\begin{array}{c}\text { GU } \\
\text { Name }\end{array}$ & $\begin{array}{l}\text { GU } \\
\text { Type }\end{array}$ & $\begin{array}{l}\text { Area } \\
\left(\mathbf{k m}^{2}\right)\end{array}$ & $\begin{array}{c}\text { Related } \\
\mathbf{G b}\end{array}$ \\
\hline 5-21.64 & NORTH AMERICAN & Groundwater Basin & $1,377.1$ & $5-21.64$ \\
\hline $5-21.65$ & SOUTH AMERICAN & Groundwater Basin & $1,002.9$ & $5-21.65$ \\
\hline 5-21.66 & SOLANO & Groundwater Basin & $1,720.1$ & $5-21.66$ \\
\hline $5-21.67$ & YOLO & Groundwater Basin & 913.9 & $5-21.67$ \\
\hline $5-21.68$ & CAPAY VALLEY & Groundwater Basin & 101.1 & $5-21.68$ \\
\hline $5-22.01$ & EASTERN SAN JOAQUIN & Groundwater Basin & $2,861.8$ & $5-22.01$ \\
\hline $5-22.02$ & MODESTO & Groundwater Basin & 997.5 & $5-22.02$ \\
\hline $5-22.03$ & TURLOCK & Groundwater Basin & $1,404.6$ & $5-22.03$ \\
\hline $5-22.04$ & MERCED & Groundwater Basin & $1,987.2$ & $5-22.04$ \\
\hline $5-22.05$ & CHOWCHILLA & Groundwater Basin & 644.4 & $5-22.05$ \\
\hline $5-22.06$ & MADERA & Groundwater Basin & $1,590.8$ & $5-22.06$ \\
\hline $5-22.07$ & DELTA-MENDOTA & Groundwater Basin & $3,021.3$ & $5-22.07$ \\
\hline $5-22.08$ & KINGS & Groundwater Basin & $3,949.0$ & $5-22.08$ \\
\hline $5-22.09$ & WESTSIDE & Groundwater Basin & $2,590.3$ & $5-22.09$ \\
\hline $5-22.10$ & PLEASANT VALLEY & Groundwater Basin & 589.5 & $5-22.10$ \\
\hline $5-22.11$ & KAWEAH & Groundwater Basin & $1,802.6$ & $5-22.11$ \\
\hline $5-22.12$ & TULARE LAKE & Groundwater Basin & $2,119.9$ & $5-22.12$ \\
\hline $5-22.13$ & TULE & Groundwater Basin & $1,898.0$ & $5-22.13$ \\
\hline $5-22.14$ & KERN COUNTY & Groundwater Basin & $7,871.9$ & $5-22.14$ \\
\hline $5-22.15$ & TRACY & Groundwater Basin & $1,396.2$ & $5-22.15$ \\
\hline $5-22.16$ & COSUMNES & Groundwater Basin & $1,135.3$ & $5-22.16$ \\
\hline $5-23$ & PANOCHE VALLEY & Groundwater Basin & 133.9 & $5-23$ \\
\hline 5-25 & KERN RIVER VALLEY & Groundwater Basin & 321.3 & $5-25$ \\
\hline 5-26 & WALKER BASIN CREEK VALLEY & Groundwater Basin & 31.0 & $5-26$ \\
\hline $5-27$ & CUMMINGS VALLEY & Groundwater Basin & 40.5 & $5-27$ \\
\hline $5-28$ & TEHACHAPI VALLEY WEST & Groundwater Basin & 72.7 & $5-28$ \\
\hline $5-29$ & CASTAC LAKE VALLEY & Groundwater Basin & 14.4 & $5-29$ \\
\hline $5-3$ & JESS VALLEY & Groundwater Basin & 27.1 & $5-3$ \\
\hline 5-30 & LOWER LAKE VALLEY & Groundwater Basin & 9.7 & 5-30 \\
\hline $5-31$ & LONG VALLEY & Groundwater Basin & 11.3 & $5-31$ \\
\hline 5-35 & MCCLOUD AREA & Groundwater Basin & 86.3 & 5-35 \\
\hline 5-36 & ROUND VALLEY & Groundwater Basin & 29.4 & 5-36 \\
\hline $5-37$ & TOAD WELL AREA & Groundwater Basin & 13.6 & $5-37$ \\
\hline $5-38$ & PONDOSA TOWN AREA & Groundwater Basin & 8.4 & $5-38$ \\
\hline $5-4$ & BIG VALLEY & Groundwater Basin & 372.6 & $5-4$ \\
\hline $5-40$ & HOT SPRINGS VALLEY & Groundwater Basin & 9.7 & $5-40$ \\
\hline $5-41$ & EGG LAKE VALLEY & Groundwater Basin & 16.6 & $5-41$ \\
\hline $5-43$ & ROCK PRAIRIE VALLEY & Groundwater Basin & 23.2 & $5-43$ \\
\hline
\end{tabular}


Table 1. California Groundwater Units.-Continued

[GU ID is the unique groundwater unit identifier. GU name is the groundwater unit name. GU names in uppercase are the same names as those used in CDWR 2002 groundwater basin map publication except for the word "HIGHLANDS" when appended. GU type identifies one of three types of groundwater units: Groundwater basin, Highland area - Gb (groundwater-basin-based highland), or Highland area - Province (province-based highland). Related Gb references the basin-ID or subbasin-ID in the CDWR 2002 groundwater basin map publication. CDWR, California Department of Water Resources; km², square kilometer; —, no data]

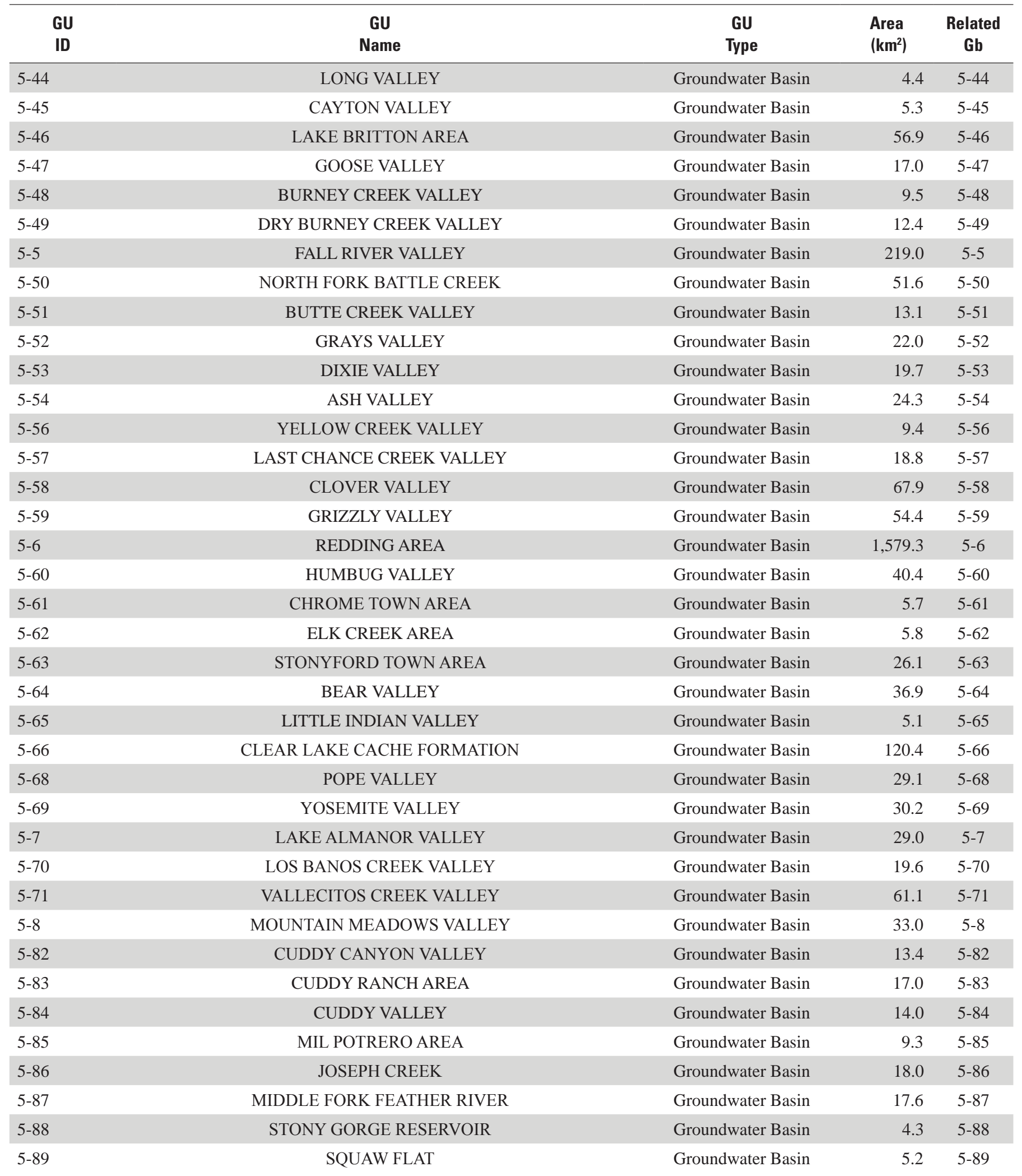


Table 1. California Groundwater Units.-Continued

[GU ID is the unique groundwater unit identifier. GU name is the groundwater unit name. GU names in uppercase are the same names as those used in CDWR 2002 groundwater basin map publication except for the word "HIGHLANDS" when appended. GU type identifies one of three types of groundwater units: Groundwater basin, Highland area - Gb (groundwater-basin-based highland), or Highland area - Province (province-based highland). Related Gb references the basin-ID or subbasin-ID in the CDWR 2002 groundwater basin map publication. CDWR, California Department of Water Resources; km², square kilometer; —, no data]

\begin{tabular}{|c|c|c|c|c|}
\hline $\begin{array}{l}\text { GU } \\
\text { ID }\end{array}$ & $\begin{array}{c}\text { GU } \\
\text { Name }\end{array}$ & $\begin{array}{l}\text { GU } \\
\text { Type }\end{array}$ & $\begin{array}{l}\text { Area } \\
\left(\mathbf{k m}^{2}\right)\end{array}$ & $\begin{array}{c}\text { Related } \\
\text { Gb }\end{array}$ \\
\hline 5-9 & INDIAN VALLEY & Groundwater Basin & 119.0 & $5-9$ \\
\hline $5-90$ & FUNKS CREEK & Groundwater Basin & 12.2 & $5-90$ \\
\hline 5-91 & ANTELOPE CREEK & Groundwater Basin & 8.3 & $5-91$ \\
\hline $5-92$ & BLANCHARD VALLEY & Groundwater Basin & 9.0 & $5-92$ \\
\hline $5-93$ & NORTH FORK CACHE CREEK & Groundwater Basin & 14.1 & $5-93$ \\
\hline $5-94$ & MIDDLE CREEK & Groundwater Basin & 2.9 & $5-94$ \\
\hline 5-95 & MEADOW VALLEY & Groundwater Basin & 23.2 & $5-95$ \\
\hline $6-1$ & SURPRISE VALLEY & Groundwater Basin & 924.0 & $6-1$ \\
\hline $6-10$ & ADOBE LAKE VALLEY & Groundwater Basin & 161.3 & $6-10$ \\
\hline $6-100$ & SECRET VALLEY & Groundwater Basin & 136.2 & $6-100$ \\
\hline 6-101 & BULL FLAT & Groundwater Basin & 73.4 & $6-101$ \\
\hline 6-104 & LONG VALLEY & Groundwater Basin & 189.4 & $6-104$ \\
\hline $6-105$ & SLINKARD VALLEY & Groundwater Basin & 18.3 & $6-105$ \\
\hline 6-106 & LITTLE ANTELOPE VALLEY & Groundwater Basin & 10.1 & $6-106$ \\
\hline 6-107 & SWEETWATER FLAT & Groundwater Basin & 19.2 & $6-107$ \\
\hline 6-11 & LONG VALLEY & Groundwater Basin & 290.7 & $6-11$ \\
\hline $6-12$ & OWENS VALLEY & Groundwater Basin & $2,675.0$ & $6-12$ \\
\hline $6-13$ & BLACK SPRINGS VALLEY & Groundwater Basin & 124.5 & $6-13$ \\
\hline $6-14$ & FISH LAKE VALLEY & Groundwater Basin & 194.8 & $6-14$ \\
\hline $6-15$ & DEEP SPRINGS VALLEY & Groundwater Basin & 121.1 & $6-15$ \\
\hline $6-16$ & EUREKA VALLEY & Groundwater Basin & 521.1 & $6-16$ \\
\hline $6-17$ & SALINE VALLEY & Groundwater Basin & 591.6 & $6-17$ \\
\hline $6-18$ & DEATH VALLEY & Groundwater Basin & $3,725.2$ & $6-18$ \\
\hline $6-19$ & WINGATE VALLEY & Groundwater Basin & 288.5 & $6-19$ \\
\hline $6-2$ & MADELINE PLAINS & Groundwater Basin & 631.7 & $6-2$ \\
\hline $6-20$ & MIDDLE AMARGOSA VALLEY & Groundwater Basin & $1,577.2$ & $6-20$ \\
\hline $6-21$ & LOWER KINGSTON VALLEY & Groundwater Basin & 970.2 & $6-21$ \\
\hline $6-22$ & UPPER KINGSTON VALLEY & Groundwater Basin & 715.3 & $6-22$ \\
\hline $6-23$ & RIGGS VALLEY & Groundwater Basin & 354.2 & $6-23$ \\
\hline $6-24$ & RED PASS VALLEY & Groundwater Basin & 389.8 & $6-24$ \\
\hline $6-25$ & BICYCLE VALLEY & Groundwater Basin & 362.0 & $6-25$ \\
\hline $6-26$ & AVAWATZ VALLEY & Groundwater Basin & 111.7 & $6-26$ \\
\hline $6-27$ & LEACH VALLEY & Groundwater Basin & 247.6 & $6-27$ \\
\hline $6-28$ & PAHRUMP VALLEY & Groundwater Basin & 376.0 & $6-28$ \\
\hline $6-29$ & MESQUITE VALLEY & Groundwater Basin & 356.6 & $6-29$ \\
\hline $6-3$ & WILLOW CREEK VALLEY & Groundwater Basin & 47.3 & $6-3$ \\
\hline $6-30$ & IVANPAH VALLEY & Groundwater Basin & 801.2 & $6-30$ \\
\hline 6-31 & KELSO VALLEY & Groundwater Basin & $1,030.7$ & $6-31$ \\
\hline
\end{tabular}


Table 1. California Groundwater Units.-Continued

[GU ID is the unique groundwater unit identifier. GU name is the groundwater unit name. GU names in uppercase are the same names as those used in CDWR 2002 groundwater basin map publication except for the word "HIGHLANDS" when appended. GU type identifies one of three types of groundwater units: Groundwater basin, Highland area - Gb (groundwater-basin-based highland), or Highland area - Province (province-based highland). Related Gb references the basin-ID or subbasin-ID in the CDWR 2002 groundwater basin map publication. CDWR, California Department of Water Resources; km², square kilometer; —, no data]

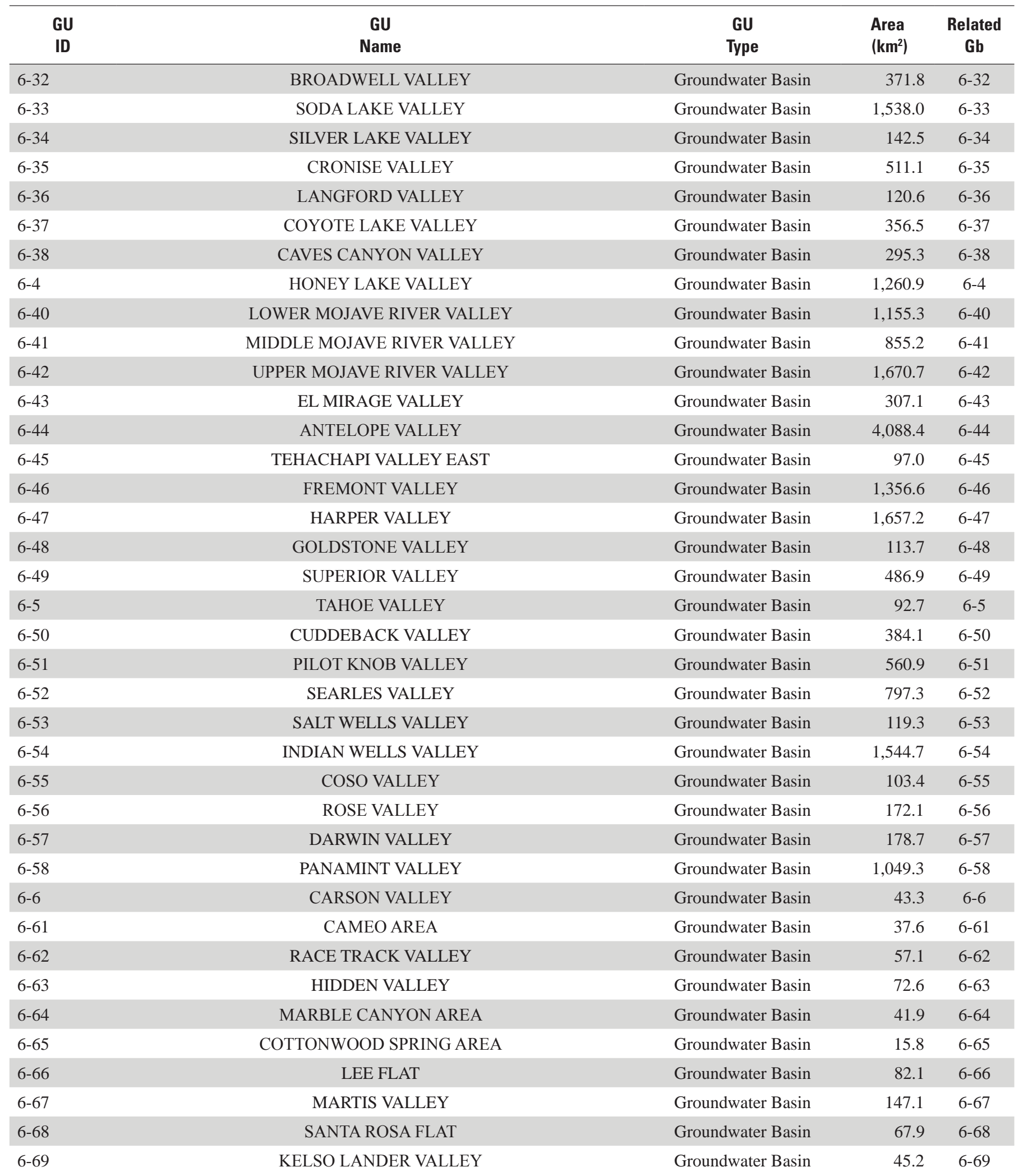


Table 1. California Groundwater Units.-Continued

[GU ID is the unique groundwater unit identifier. GU name is the groundwater unit name. GU names in uppercase are the same names as those used in CDWR 2002 groundwater basin map publication except for the word "HIGHLANDS" when appended. GU type identifies one of three types of groundwater units: Groundwater basin, Highland area - Gb (groundwater-basin-based highland), or Highland area - Province (province-based highland). Related Gb references the basin-ID or subbasin-ID in the CDWR 2002 groundwater basin map publication. CDWR, California Department of Water Resources; km², square kilometer; -, no data]

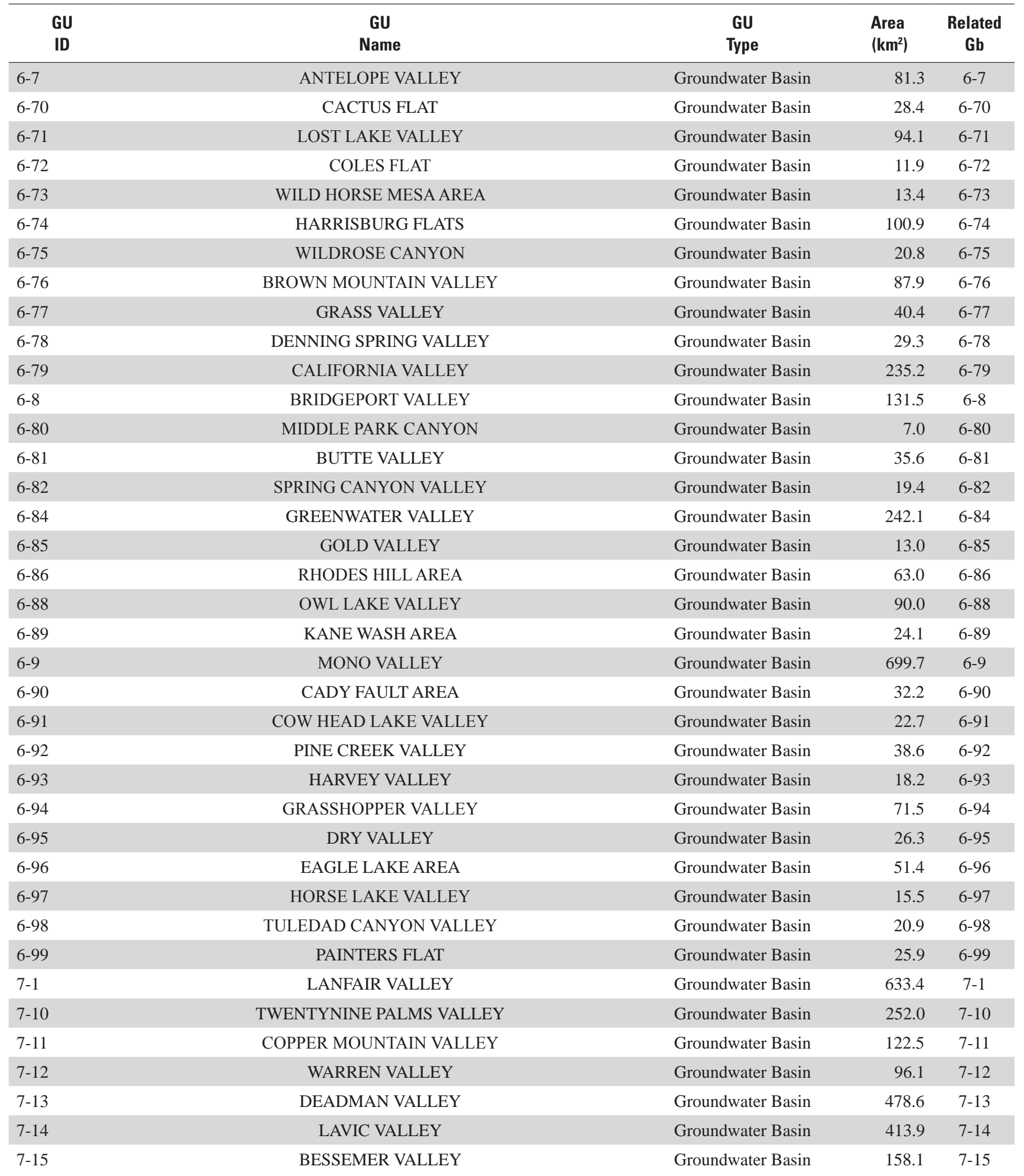


Table 1. California Groundwater Units.—Continued

[GU ID is the unique groundwater unit identifier. GU name is the groundwater unit name. GU names in uppercase are the same names as those used in CDWR 2002 groundwater basin map publication except for the word "HIGHLANDS" when appended. GU type identifies one of three types of groundwater units: Groundwater basin, Highland area - Gb (groundwater-basin-based highland), or Highland area - Province (province-based highland). Related Gb references the basin-ID or subbasin-ID in the CDWR 2002 groundwater basin map publication. CDWR, California Department of Water Resources; km², square kilometer; —, no data]

\begin{tabular}{|c|c|c|c|c|}
\hline $\begin{array}{l}\text { GU } \\
\text { ID }\end{array}$ & $\begin{array}{l}\text { GU } \\
\text { Name }\end{array}$ & $\begin{array}{l}\text { GU } \\
\text { Type }\end{array}$ & $\begin{array}{l}\text { Area } \\
\left(\mathbf{k m}^{2}\right)\end{array}$ & $\begin{array}{c}\text { Related } \\
\text { Gb }\end{array}$ \\
\hline $7-16$ & AMES VALLEY & Groundwater Basin & 438.8 & $7-16$ \\
\hline $7-17$ & MEANS VALLEY & Groundwater Basin & 60.5 & $7-17$ \\
\hline $7-18$ & JOHNSON VALLEY & Groundwater Basin & 453.5 & $7-18$ \\
\hline 7-19 & LUCERNE VALLEY & Groundwater Basin & 596.6 & $7-19$ \\
\hline $7-2$ & FENNER VALLEY & Groundwater Basin & $1,831.1$ & $7-2$ \\
\hline $7-20$ & MORONGO VALLEY & Groundwater Basin & 29.3 & $7-20$ \\
\hline $7-21$ & COACHELLA VALLEY & Groundwater Basin & $1,963.6$ & $7-21$ \\
\hline $7-22$ & WEST SALTON SEA & Groundwater Basin & 426.5 & $7-22$ \\
\hline $7-24$ & BORREGO VALLEY & Groundwater Basin & 617.4 & $7-24$ \\
\hline 7-25 & OCOTILLO-CLARK VALLEY & Groundwater Basin & 899.5 & $7-25$ \\
\hline $7-26$ & TERWILLIGER VALLEY & Groundwater Basin & 32.4 & $7-26$ \\
\hline $7-27$ & SAN FELIPE VALLEY & Groundwater Basin & 94.6 & $7-27$ \\
\hline $7-28$ & VALLECITO-CARRIZO VALLEY & Groundwater Basin & 493.0 & $7-28$ \\
\hline 7-29 & COYOTE WELLS VALLEY & Groundwater Basin & 589.4 & $7-29$ \\
\hline 7-3 & WARD VALLEY & Groundwater Basin & $2,256.5$ & $7-3$ \\
\hline 7-30 & IMPERIAL VALLEY & Groundwater Basin & $3,876.4$ & $7-30$ \\
\hline 7-31 & OROCOPIA VALLEY & Groundwater Basin & 389.4 & $7-31$ \\
\hline 7-32 & CHOCOLATE VALLEY & Groundwater Basin & 522.5 & $7-32$ \\
\hline 7-33 & EAST SALTON SEA & Groundwater Basin & 788.5 & $7-33$ \\
\hline 7-34 & AMOS VALLEY & Groundwater Basin & 525.8 & $7-34$ \\
\hline $7-35$ & OGILBY VALLEY & Groundwater Basin & 539.0 & $7-35$ \\
\hline $7-36$ & YUMA VALLEY & Groundwater Basin & 501.6 & $7-36$ \\
\hline $7-37$ & ARROYO SECO VALLEY & Groundwater Basin & $1,038.1$ & $7-37$ \\
\hline 7-38 & PALO VERDE VALLEY & Groundwater Basin & 295.3 & $7-38$ \\
\hline 7-39 & PALO VERDE MESA & Groundwater Basin & 910.2 & 7-39 \\
\hline $7-4$ & RICE VALLEY & Groundwater Basin & 761.1 & $7-4$ \\
\hline $7-40$ & QUIEN SABE POINT VALLEY & Groundwater Basin & 101.7 & $7-40$ \\
\hline $7-41$ & CALZONA VALLEY & Groundwater Basin & 325.9 & $7-41$ \\
\hline $7-42$ & VIDAL VALLEY & Groundwater Basin & 557.1 & $7-42$ \\
\hline $7-43$ & CHEMEHUEVI VALLEY & Groundwater Basin & $1,100.5$ & $7-43$ \\
\hline $7-44$ & NEEDLES VALLEY & Groundwater Basin & 355.7 & $7-44$ \\
\hline $7-45$ & PIUTE VALLEY & Groundwater Basin & 708.6 & $7-45$ \\
\hline $7-46$ & CANEBRAKE VALLEY & Groundwater Basin & 21.9 & $7-46$ \\
\hline $7-47$ & JACUMBA VALLEY & Groundwater Basin & 9.9 & $7-47$ \\
\hline $7-48$ & HELENDALE FAULT VALLEY & Groundwater Basin & 10.6 & $7-48$ \\
\hline 7-49 & PIPES CANYON FAULT VALLEY & Groundwater Basin & 13.7 & $7-49$ \\
\hline 7-5 & CHUCKWALLA VALLEY & Groundwater Basin & $2,434.5$ & $7-5$ \\
\hline $7-50$ & IRON RIDGE AREA & Groundwater Basin & 21.2 & $7-50$ \\
\hline
\end{tabular}


Table 1. California Groundwater Units.-Continued

[GU ID is the unique groundwater unit identifier. GU name is the groundwater unit name. GU names in uppercase are the same names as those used in CDWR 2002 groundwater basin map publication except for the word "HIGHLANDS" when appended. GU type identifies one of three types of groundwater units: Groundwater basin, Highland area - Gb (groundwater-basin-based highland), or Highland area - Province (province-based highland). Related Gb references the basin-ID or subbasin-ID in the CDWR 2002 groundwater basin map publication. CDWR, California Department of Water Resources; km², square kilometer; —, no data]

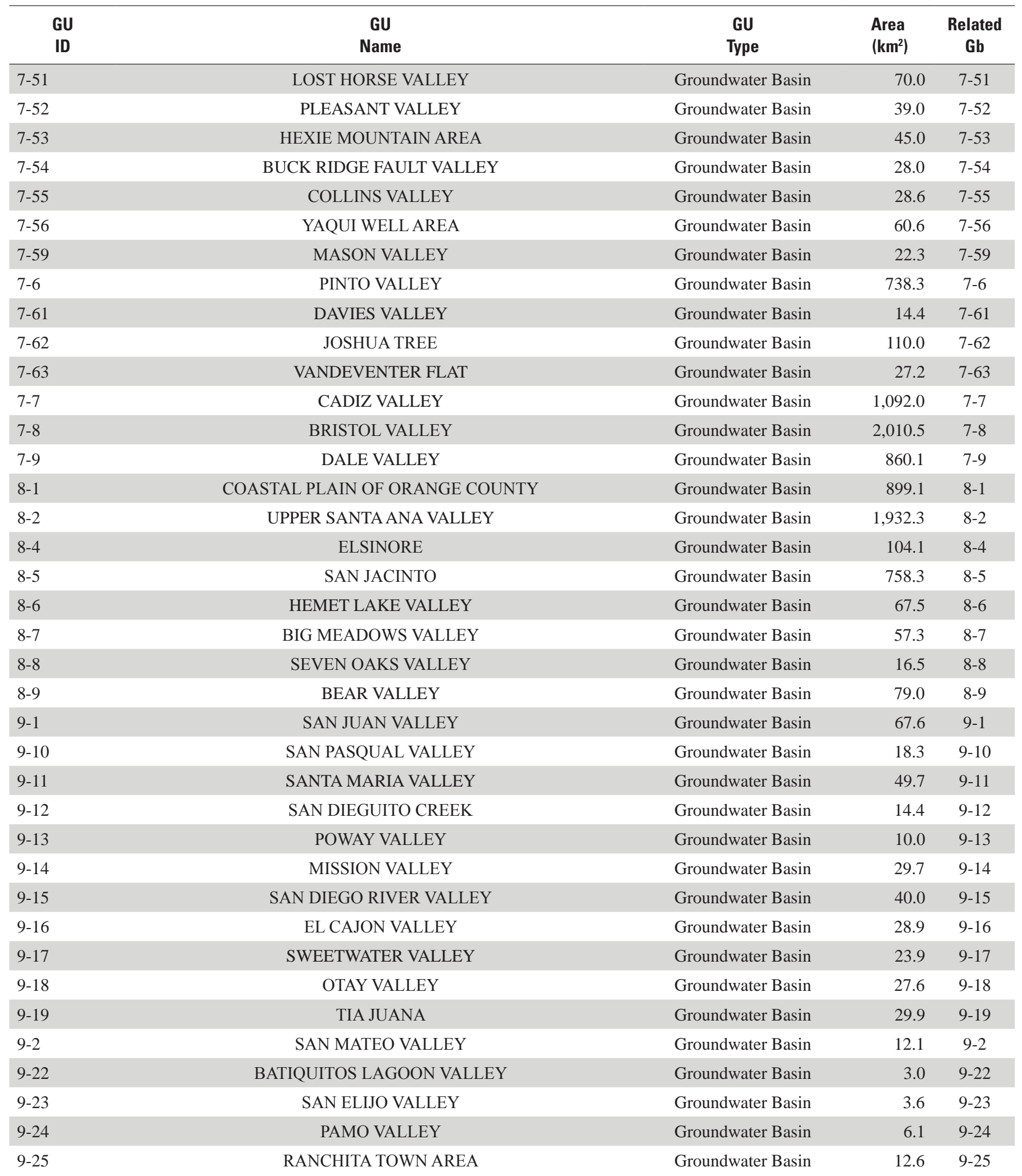


Table 1. California Groundwater Units.-Continued

[GU ID is the unique groundwater unit identifier. GU name is the groundwater unit name. GU names in uppercase are the same names as those used in CDWR 2002 groundwater basin map publication except for the word "HIGHLANDS" when appended. GU type identifies one of three types of groundwater units: Groundwater basin, Highland area - Gb (groundwater-basin-based highland), or Highland area - Province (province-based highland). Related Gb references the basin-ID or subbasin-ID in the CDWR 2002 groundwater basin map publication. CDWR, California Department of Water Resources; km², square kilometer; —, no data]

\begin{tabular}{|c|c|c|c|c|}
\hline $\begin{array}{l}\text { GU } \\
\text { ID }\end{array}$ & $\begin{array}{c}\text { GU } \\
\text { Name }\end{array}$ & $\begin{array}{l}\text { GU } \\
\text { Type }\end{array}$ & $\begin{array}{l}\text { Area } \\
\left(\mathbf{k m}^{2}\right)\end{array}$ & $\begin{array}{c}\text { Related } \\
\text { Gb }\end{array}$ \\
\hline $9-27$ & COTTONWOOD VALLEY & Groundwater Basin & 15.5 & $9-27$ \\
\hline $9-28$ & CAMPO VALLEY & Groundwater Basin & 14.3 & $9-28$ \\
\hline $9-29$ & POTRERO VALLEY & Groundwater Basin & 8.2 & $9-29$ \\
\hline $9-3$ & SAN ONOFRE VALLEY & Groundwater Basin & 5.1 & $9-3$ \\
\hline $9-32$ & SAN MARCOS AREA & Groundwater Basin & 8.6 & $9-32$ \\
\hline $9-4$ & SANTA MARGARITA VALLEY & Groundwater Basin & 32.2 & $9-4$ \\
\hline $9-5$ & TEMECULA VALLEY & Groundwater Basin & 355.1 & $9-5$ \\
\hline $9-6$ & CAHUILLA VALLEY & Groundwater Basin & 73.7 & $9-6$ \\
\hline $9-7$ & SAN LUIS REY VALLEY & Groundwater Basin & 120.1 & $9-7$ \\
\hline $9-8$ & WARNER VALLEY & Groundwater Basin & 97.0 & $9-8$ \\
\hline $9-9$ & ESCONDIDO VALLEY & Groundwater Basin & 11.7 & $9-9$ \\
\hline- & Subtotal & 463 & 160,977 & - \\
\hline Hgb-1-1 & SMITH RIVER PLAIN HIGHLANDS & Highland area - Gb & $1,528.8$ & $1-1$ \\
\hline Hgb-1-10 & EEL RIVER VALLEY HIGHLANDS & Highland area - Gb & 917.9 & $1-10$ \\
\hline Hgb-1-11 & COVELO ROUND VALLEY HIGHLANDS & Highland area - Gb & 145.1 & $1-11$ \\
\hline Hgb-1-12 & LAYTONVILLE VALLEY HIGHLANDS & Highland area - Gb & 64.6 & $1-12$ \\
\hline Hgb-1-13 & LITTLE LAKE VALLEY HIGHLANDS & Highland area - Gb & 119.1 & $1-13$ \\
\hline Hgb-1-14 & LOWER KLAMATH RIVER VALLEY HIGHLANDS & Highland area - Gb & $5,022.4$ & $1-14$ \\
\hline Hgb-1-15 & HAPPY CAMP TOWN AREA HIGHLANDS & Highland area - Gb & 891.6 & $1-15$ \\
\hline Hgb-1-16 & SEIAD VALLEY HIGHLANDS & Highland area - Gb & $2,600.5$ & $1-16$ \\
\hline Hgb-1-17 & BRAY TOWN AREA HIGHLANDS & Highland area - Gb & 304.9 & $1-17$ \\
\hline Hgb-1-18 & RED ROCK VALLEY HIGHLANDS & Highland area - Gb & 201.5 & $1-18$ \\
\hline Hgb-1-19 & ANDERSON VALLEY HIGHLANDS & Highland area - Gb & 457.8 & $1-19$ \\
\hline Hgb-1-2 & KLAMATH RIVER VALLEY HIGHLANDS & Highland area - Gb & $2,399.3$ & $1-2$ \\
\hline Hgb-1-20 & GARCIA RIVER VALLEY HIGHLANDS & Highland area - Gb & 17.9 & $1-20$ \\
\hline Hgb-1-21 & FORT BRAGG TERRACE AREA HIGHLANDS & Highland area - Gb & 798.3 & $1-21$ \\
\hline Hgb-1-22 & FAIRCHILD SWAMP VALLEY HIGHLANDS & Highland area - Gb & 63.7 & $1-22$ \\
\hline Hgb-1-25 & PRAIRIE CREEK AREA HIGHLANDS & Highland area - Gb & 12.7 & $1-25$ \\
\hline Hgb-1-26 & REDWOOD CREEK AREA HIGHLANDS & Highland area - Gb & 658.7 & $1-26$ \\
\hline Hgb-1-27 & BIG LAGOON AREA HIGHLANDS & Highland area - Gb & 128.9 & $1-27$ \\
\hline Hgb-1-28 & MATTOLE RIVER VALLEY HIGHLANDS & Highland area - Gb & 234.4 & $1-28$ \\
\hline Hgb-1-29 & HONEYDEW TOWN AREA HIGHLANDS & Highland area - Gb & 480.2 & $1-29$ \\
\hline Hgb-1-3 & BUTTE VALLEY HIGHLANDS & Highland area - Gb & 641.7 & $1-3$ \\
\hline Hgb-1-30 & PEPPERWOOD TOWN AREA HIGHLANDS & Highland area - Gb & 333.1 & $1-30$ \\
\hline Hgb-1-31 & WEOTT TOWN AREA HIGHLANDS & Highland area - Gb & $5,599.0$ & $1-31$ \\
\hline Hgb-1-32 & GARBERVILLE TOWN AREA HIGHLANDS & Highland area - Gb & $1,093.6$ & $1-32$ \\
\hline Hgb-1-33 & LARABEE VALLEY HIGHLANDS & Highland area - Gb & 29.4 & $1-33$ \\
\hline Hgb-1-34 & DINSMORES TOWN AREA HIGHLANDS & Highland area - Gb & 183.6 & $1-34$ \\
\hline
\end{tabular}


Table 1. California Groundwater Units.-Continued

[GU ID is the unique groundwater unit identifier. GU name is the groundwater unit name. GU names in uppercase are the same names as those used in CDWR 2002 groundwater basin map publication except for the word "HIGHLANDS" when appended. GU type identifies one of three types of groundwater units: Groundwater basin, Highland area - Gb (groundwater-basin-based highland), or Highland area - Province (province-based highland). Related Gb references the basin-ID or subbasin-ID in the CDWR 2002 groundwater basin map publication. CDWR, California Department of Water Resources; km², square kilometer; —, no data]

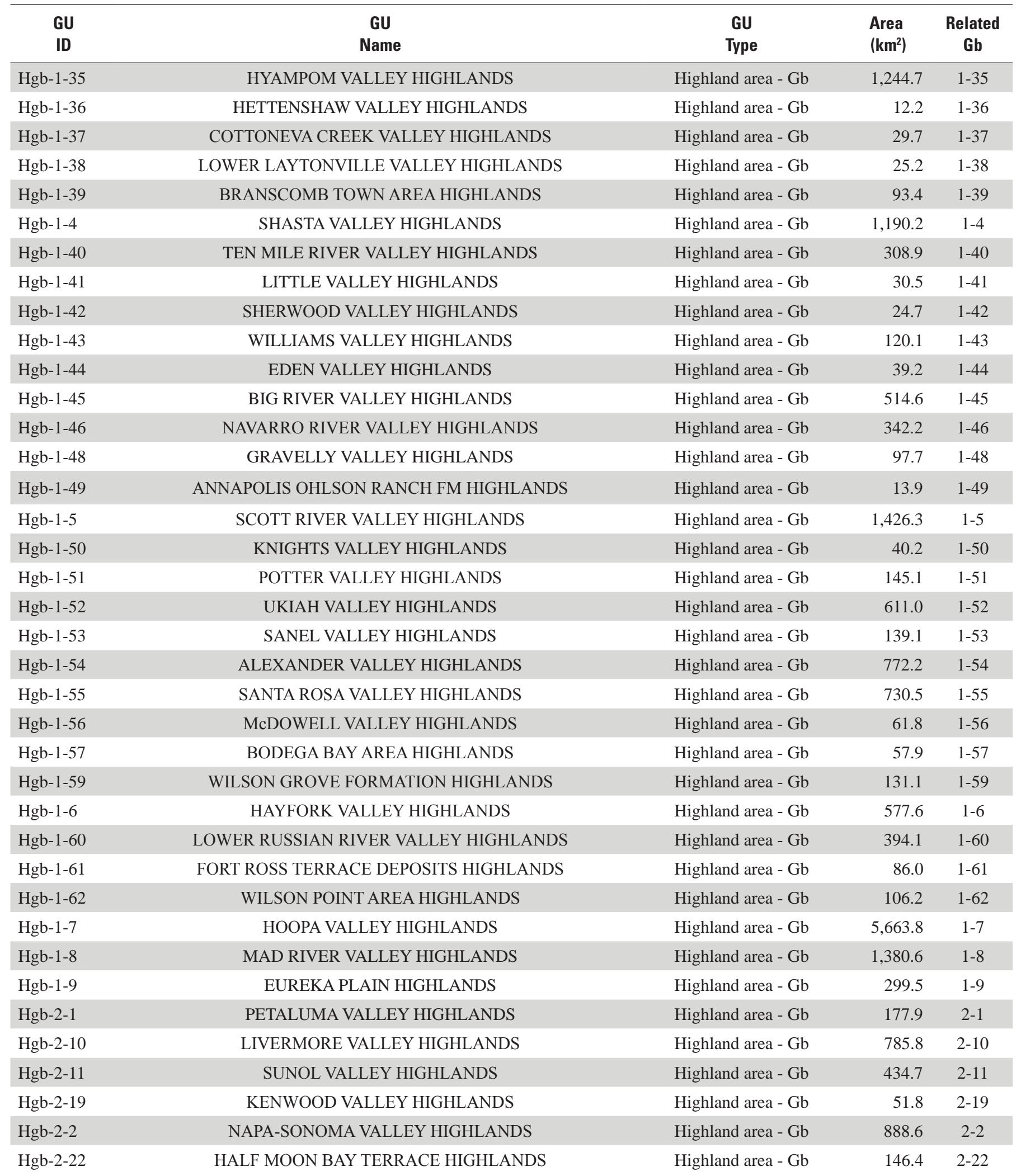


Table 1. California Groundwater Units.—Continued

[GU ID is the unique groundwater unit identifier. GU name is the groundwater unit name. GU names in uppercase are the same names as those used in CDWR 2002 groundwater basin map publication except for the word "HIGHLANDS" when appended. GU type identifies one of three types of groundwater units: Groundwater basin, Highland area - Gb (groundwater-basin-based highland), or Highland area - Province (province-based highland). Related Gb references the basin-ID or subbasin-ID in the CDWR 2002 groundwater basin map publication. CDWR, California Department of Water Resources; km², square kilometer; —, no data]

\begin{tabular}{|c|c|c|c|c|}
\hline $\begin{array}{l}\text { GU } \\
\text { ID }\end{array}$ & $\begin{array}{c}\text { GU } \\
\text { Name }\end{array}$ & $\begin{array}{l}\text { GU } \\
\text { Type }\end{array}$ & $\begin{array}{l}\text { Area } \\
\left(\mathbf{k m}^{2}\right)\end{array}$ & $\begin{array}{c}\text { Related } \\
\text { Gb }\end{array}$ \\
\hline Hgb-2-24 & SAN GREGORIO VALLEY HIGHLANDS & Highland area - Gb & 126.7 & $2-24$ \\
\hline Hgb-2-26 & PESCADERO VALLEY HIGHLANDS & Highland area - Gb & 198.4 & $2-26$ \\
\hline Hgb-2-27 & SAND POINT AREA HIGHLANDS & Highland area - Gb & 0.5 & $2-27$ \\
\hline Hgb-2-28 & ROSS VALLEY HIGHLANDS & Highland area - Gb & 75.9 & $2-28$ \\
\hline Hgb-2-29 & SAN RAFAEL VALLEY HIGHLANDS & Highland area - Gb & 17.1 & $2-29$ \\
\hline Hgb-2-3 & SUISUN-FAIRFIELD VALLEY HIGHLANDS & Highland area - Gb & 335.1 & $2-3$ \\
\hline Hgb-2-30 & NOVATO VALLEY HIGHLANDS & Highland area - Gb & 149.6 & $2-30$ \\
\hline Hgb-2-31 & ARROYO DEL HAMBRE VALLEY HIGHLANDS & Highland area - Gb & 39.4 & $2-31$ \\
\hline Hgb-2-35 & WESTSIDE HIGHLANDS & Highland area - Gb & 33.0 & $2-35$ \\
\hline Hgb-2-36 & SAN PEDRO VALLEY HIGHLANDS & Highland area - Gb & 19.5 & $2-36$ \\
\hline Hgb-2-4 & PITTSBURG PLAIN HIGHLANDS & Highland area - Gb & 57.5 & $2-4$ \\
\hline Hgb-2-5 & CLAYTON VALLEY HIGHLANDS & Highland area - Gb & 70.7 & $2-5$ \\
\hline Hgb-2-6 & YGNACIO VALLEY HIGHLANDS & Highland area - Gb & 165.0 & $2-6$ \\
\hline Hgb-2-7 & SAN RAMON VALLEY HIGHLANDS & Highland area - Gb & 100.7 & $2-7$ \\
\hline Hgb-2-8 & CASTRO VALLEY HIGHLANDS & Highland area - Gb & 122.2 & $2-8$ \\
\hline Hgb-2-9 & SANTA CLARA VALLEY HIGHLANDS & Highland area - Gb & $1,395.3$ & $2-9$ \\
\hline Hgb-3-12 & SANTA MARIA HIGHLANDS & Highland area - Gb & $2,122.4$ & $3-12$ \\
\hline Hgb-3-13 & CUYAMA VALLEY HIGHLANDS & Highland area - Gb & $1,145.2$ & $3-13$ \\
\hline Hgb-3-14 & SAN ANTONIO CREEK VALLEY HIGHLANDS & Highland area - Gb & 132.8 & $3-14$ \\
\hline Hgb-3-15 & SANTA YNEZ RIVER VALLEY HIGHLANDS & Highland area - Gb & $1,644.5$ & 3-15 \\
\hline Hgb-3-16 & GOLETA HIGHLANDS & Highland area - Gb & 96.1 & $3-16$ \\
\hline Hgb-3-17 & SANTA BARBARA HIGHLANDS & Highland area - Gb & 9.9 & $3-17$ \\
\hline Hgb-3-18 & CARPINTERIA HIGHLANDS & Highland area - Gb & 86.7 & $3-18$ \\
\hline Hgb-3-19 & CARRIZO PLAIN HIGHLANDS & Highland area - Gb & 305.4 & $3-19$ \\
\hline Hgb-3-2 & PAJARO VALLEY HIGHLANDS & Highland area - Gb & 110.3 & $3-2$ \\
\hline Hgb-3-20 & ANO NUEVO AREA HIGHLANDS & Highland area - Gb & 13.3 & $3-20$ \\
\hline Hgb-3-21 & SANTA CRUZ PURISIMA FORMATION HIGHLANDS & Highland area - Gb & 108.9 & $3-21$ \\
\hline Hgb-3-22 & SANTA ANA VALLEY HIGHLANDS & Highland area - Gb & 37.8 & $3-22$ \\
\hline Hgb-3-23 & UPPER SANTA ANA VALLEY HIGHLANDS & Highland area - Gb & 37.3 & $3-23$ \\
\hline Hgb-3-24 & QUIEN SABE VALLEY HIGHLANDS & Highland area - Gb & 79.1 & $3-24$ \\
\hline Hgb-3-25 & TRES PINOS VALLEY HIGHLANDS & Highland area - Gb & 11.3 & $3-25$ \\
\hline Hgb-3-26 & WEST SANTA CRUZ TERRACE HIGHLANDS & Highland area - Gb & 42.5 & $3-26$ \\
\hline Hgb-3-27 & SCOTTS VALLEY HIGHLANDS & Highland area - Gb & 2.5 & $3-27$ \\
\hline Hgb-3-28 & SAN BENITO RIVER VALLEY HIGHLANDS & Highland area - Gb & 264.3 & $3-28$ \\
\hline Hgb-3-29 & DRY LAKE VALLEY HIGHLANDS & Highland area - Gb & 276.2 & $3-29$ \\
\hline Hgb-3-3 & GILROY-HOLLISTER VALLEY HIGHLANDS & Highland area - Gb & $1,640.4$ & $3-3$ \\
\hline Hgb-3-30 & BITTER WATER VALLEY HIGHLANDS & Highland area - Gb & 337.7 & $3-30$ \\
\hline Hgb-3-31 & HERNANDEZ VALLEY HIGHLANDS & Highland area - Gb & 202.7 & 3-31 \\
\hline
\end{tabular}


Table 1. California Groundwater Units.-Continued

[GU ID is the unique groundwater unit identifier. GU name is the groundwater unit name. GU names in uppercase are the same names as those used in CDWR 2002 groundwater basin map publication except for the word "HIGHLANDS" when appended. GU type identifies one of three types of groundwater units: Groundwater basin, Highland area - Gb (groundwater-basin-based highland), or Highland area - Province (province-based highland). Related Gb references the basin-ID or subbasin-ID in the CDWR 2002 groundwater basin map publication. CDWR, California Department of Water Resources; km², square kilometer; -, no data]

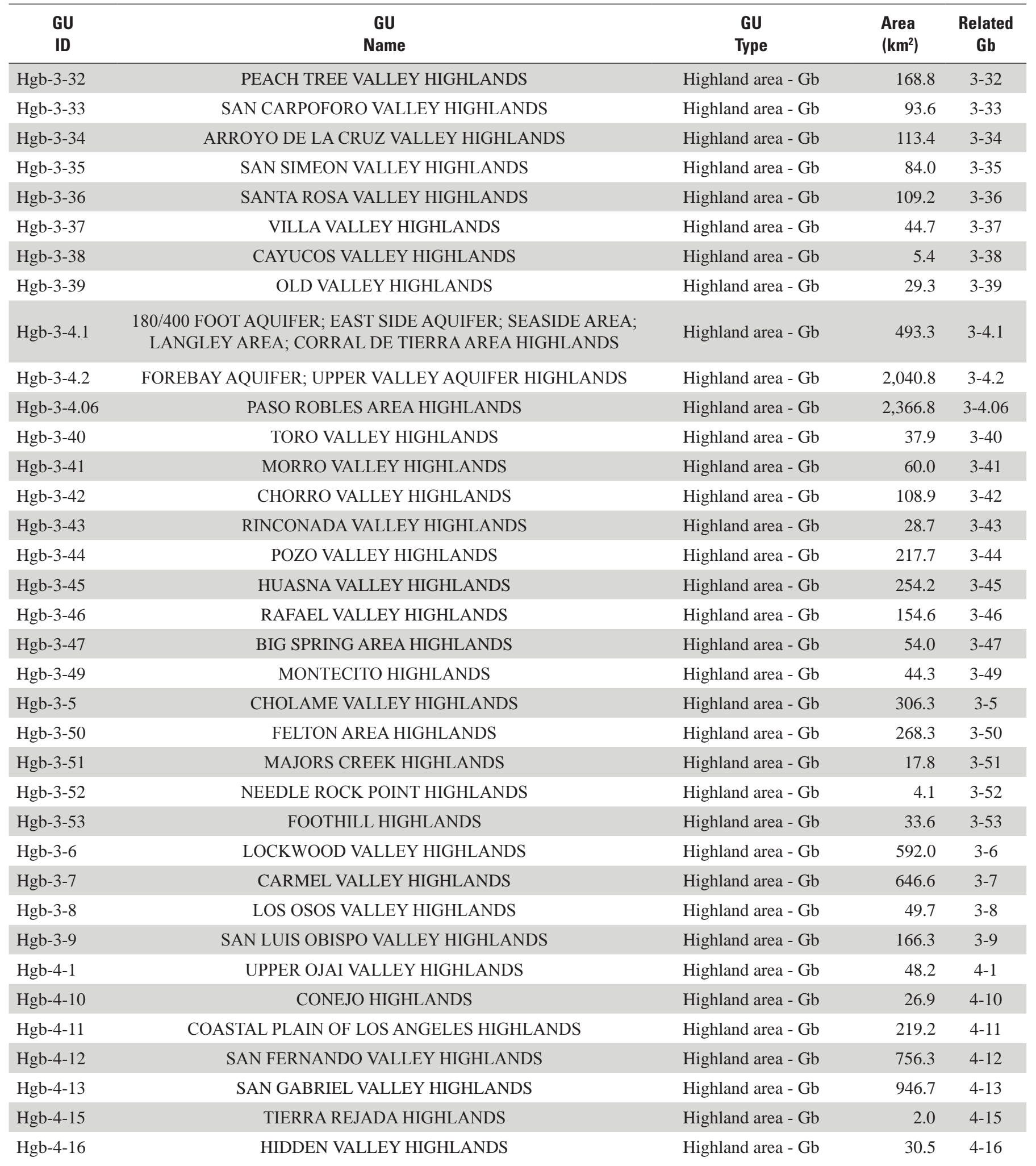


Table 1. California Groundwater Units.—Continued

[GU ID is the unique groundwater unit identifier. GU name is the groundwater unit name. GU names in uppercase are the same names as those used in CDWR 2002 groundwater basin map publication except for the word "HIGHLANDS" when appended. GU type identifies one of three types of groundwater units: Groundwater basin, Highland area - Gb (groundwater-basin-based highland), or Highland area - Province (province-based highland). Related Gb references the basin-ID or subbasin-ID in the CDWR 2002 groundwater basin map publication. CDWR, California Department of Water Resources; km², square kilometer; —, no data]

\begin{tabular}{|c|c|c|c|c|}
\hline $\begin{array}{l}\text { GU } \\
\text { ID }\end{array}$ & $\begin{array}{c}\text { GU } \\
\text { Name }\end{array}$ & $\begin{array}{l}\text { GU } \\
\text { Type }\end{array}$ & $\begin{array}{l}\text { Area } \\
\left(\mathbf{k m}^{2}\right)\end{array}$ & $\begin{array}{c}\text { Related } \\
\text { Gb }\end{array}$ \\
\hline Hgb-4-17 & LOCKWOOD VALLEY HIGHLANDS & Highland area - Gb & 91.4 & $4-17$ \\
\hline Hgb-4-18 & HUNGRY VALLEY HIGHLANDS & Highland area - Gb & 69.6 & $4-18$ \\
\hline Hgb-4-19 & THOUSAND OAKS AREA HIGHLANDS & Highland area - Gb & 31.1 & $4-19$ \\
\hline Hgb-4-2 & OJAI VALLEY HIGHLANDS & Highland area - Gb & 63.2 & $4-2$ \\
\hline Hgb-4-20 & RUSSELL VALLEY HIGHLANDS & Highland area - Gb & 44.3 & $4-20$ \\
\hline Hgb-4-22 & MALIBU VALLEY HIGHLANDS & Highland area - Gb & 157.8 & $4-22$ \\
\hline Hgb-4-23 & RAYMOND HIGHLANDS & Highland area - Gb & 141.4 & $4-23$ \\
\hline Hgb-4-3 & VENTURA RIVER VALLEY HIGHLANDS & Highland area - Gb & 420.3 & $4-3$ \\
\hline Hgb-4-4 & SANTA CLARA RIVER VALLEY HIGHLANDS & Highland area - Gb & $3,022.3$ & $4-4$ \\
\hline Hgb-4-5 & ACTON VALLEY HIGHLANDS & Highland area - Gb & 65.3 & $4-5$ \\
\hline Hgb-4-6 & PLEASANT VALLEY HIGHLANDS & Highland area - Gb & 26.2 & $4-6$ \\
\hline Hgb-4-7 & ARROYO SANTA ROSA VALLEY HIGHLANDS & Highland area - Gb & 12.4 & $4-7$ \\
\hline Hgb-4-8 & LAS POSAS VALLEY HIGHLANDS & Highland area - Gb & 82.6 & $4-8$ \\
\hline Hgb-4-9 & SIMI VALLEY HIGHLANDS & Highland area - Gb & 172.4 & $4-9$ \\
\hline Hgb-5-1 & GOOSE LAKE HIGHLANDS & Highland area - Gb & 452.2 & $5-1$ \\
\hline Hgb-5-10 & AMERICAN VALLEY HIGHLANDS & Highland area - Gb & 238.0 & $5-10$ \\
\hline Hgb-5-11 & MOHAWK VALLEY HIGHLANDS & Highland area - Gb & 226.3 & $5-11$ \\
\hline Hgb-5-12 & SIERRA VALLEY HIGHLANDS & Highland area - Gb & 905.8 & $5-12$ \\
\hline Hgb-5-13 & UPPER LAKE VALLEY HIGHLANDS & Highland area - Gb & 199.6 & $5-13$ \\
\hline Hgb-5-14 & SCOTTS VALLEY HIGHLANDS & Highland area - Gb & 156.7 & $5-14$ \\
\hline Hgb-5-15 & BIG VALLEY HIGHLANDS & Highland area - Gb & 219.7 & $5-15$ \\
\hline Hgb-5-16 & HIGH VALLEY HIGHLANDS & Highland area - Gb & 20.6 & $5-16$ \\
\hline Hgb-5-17 & BURNS VALLEY HIGHLANDS & Highland area - Gb & 8.9 & $5-17$ \\
\hline Hgb-5-18 & COYOTE VALLEY HIGHLANDS & Highland area - Gb & 142.1 & $5-18$ \\
\hline Hgb-5-19 & COLLAYOMI VALLEY HIGHLANDS & Highland area - Gb & 166.7 & $5-19$ \\
\hline Hgb-5-2 & ALTURAS AREA HIGHLANDS & Highland area - Gb & $2,085.5$ & $5-2$ \\
\hline Hgb-5-20 & BERRYESSA VALLEY HIGHLANDS & Highland area - Gb & 50.5 & $5-20$ \\
\hline Hgb-5-21.50 & RED BLUFF HIGHLANDS & Highland area - Gb & 467.3 & $5-21.50$ \\
\hline Hgb-5-21.51 & CORNING HIGHLANDS & Highland area - Gb & 729.2 & $5-21.51$ \\
\hline Hgb-5-21.52 & COLUSA HIGHLANDS & Highland area - Gb & $1,233.6$ & $5-21.52$ \\
\hline Hgb-5-21.53 & BEND HIGHLANDS & Highland area - Gb & 192.7 & $5-21.53$ \\
\hline Hgb-5-21.54 & ANTELOPE HIGHLANDS & Highland area - Gb & 410.3 & $5-21.54$ \\
\hline Hgb-5-21.55 & DYE CREEK HIGHLANDS & Highland area - Gb & 179.0 & $5-21.55$ \\
\hline Hgb-5-21.56 & LOS MOLINOS HIGHLANDS & Highland area - Gb & $1,015.9$ & $5-21.56$ \\
\hline Hgb-5-21.57 & VINA HIGHLANDS & Highland area - Gb & 666.3 & $5-21.57$ \\
\hline Hgb-5-21.58 & WEST BUTTE HIGHLANDS & Highland area - Gb & 291.2 & $5-21.58$ \\
\hline Hgb-5-21.59 & EAST BUTTE HIGHLANDS & Highland area - Gb & $4,416.5$ & $5-21.59$ \\
\hline Hgb-5-21.60 & NORTH YUBA HIGHLANDS & Highland area - Gb & 418.6 & $5-21.60$ \\
\hline
\end{tabular}


Table 1. California Groundwater Units.-Continued

[GU ID is the unique groundwater unit identifier. GU name is the groundwater unit name. GU names in uppercase are the same names as those used in CDWR 2002 groundwater basin map publication except for the word "HIGHLANDS" when appended. GU type identifies one of three types of groundwater units: Groundwater basin, Highland area - Gb (groundwater-basin-based highland), or Highland area - Province (province-based highland). Related Gb references the basin-ID or subbasin-ID in the CDWR 2002 groundwater basin map publication. CDWR, California Department of Water Resources; km², square kilometer; -, no data]

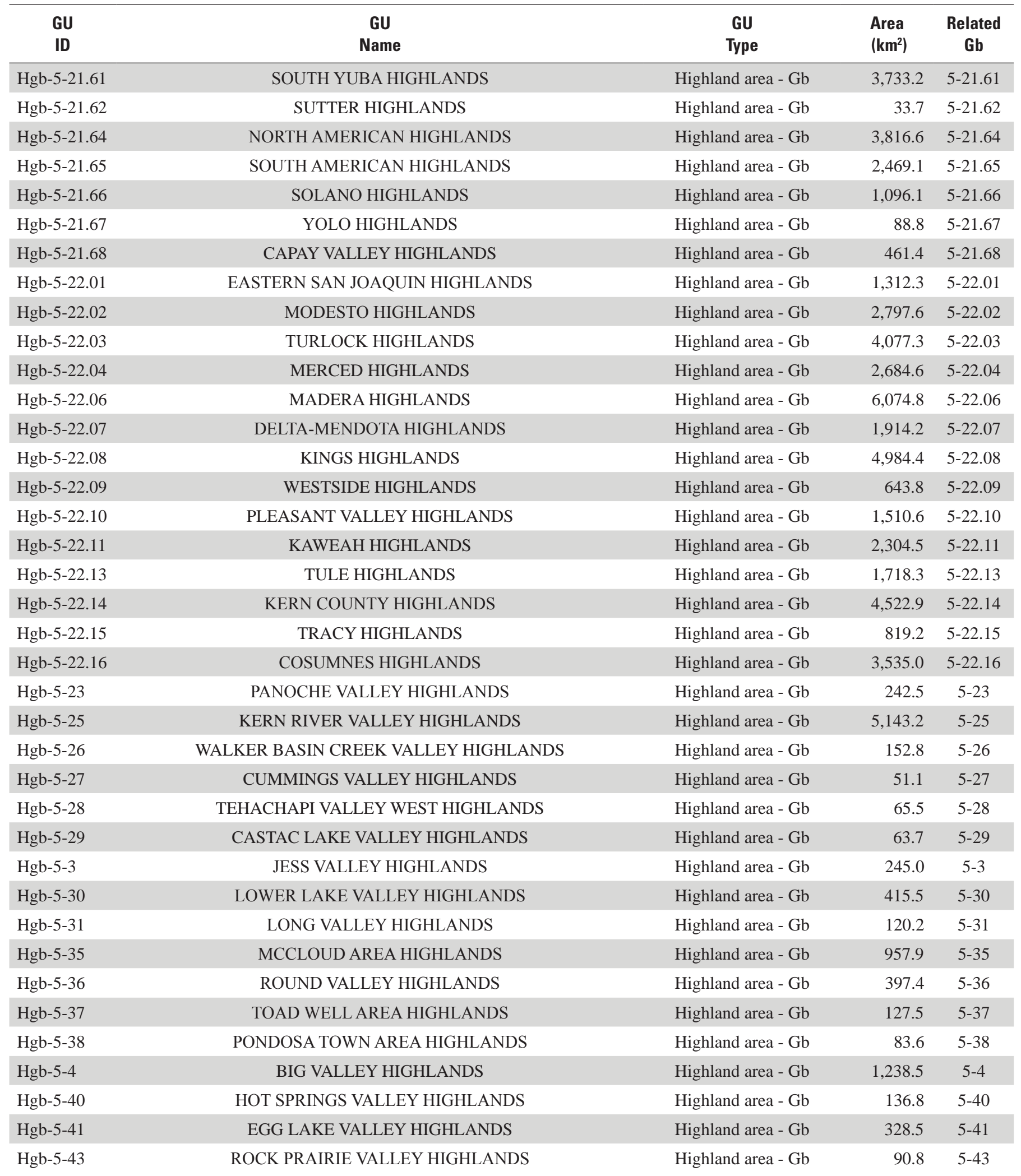


Table 1. California Groundwater Units.—Continued

[GU ID is the unique groundwater unit identifier. GU name is the groundwater unit name. GU names in uppercase are the same names as those used in CDWR 2002 groundwater basin map publication except for the word "HIGHLANDS" when appended. GU type identifies one of three types of groundwater units: Groundwater basin, Highland area - Gb (groundwater-basin-based highland), or Highland area - Province (province-based highland). Related Gb references the basin-ID or subbasin-ID in the CDWR 2002 groundwater basin map publication. CDWR, California Department of Water Resources; km², square kilometer; —, no data]

\begin{tabular}{|c|c|c|c|c|}
\hline $\begin{array}{l}\text { GU } \\
\text { ID }\end{array}$ & $\begin{array}{c}\text { GU } \\
\text { Name }\end{array}$ & $\begin{array}{l}\text { GU } \\
\text { Type }\end{array}$ & $\begin{array}{l}\text { Area } \\
\left(\mathbf{k m}^{2}\right)\end{array}$ & $\begin{array}{c}\text { Related } \\
\text { Gb }\end{array}$ \\
\hline Hgb-5-44 & LONG VALLEY HIGHLANDS & Highland area - Gb & 11.2 & $5-44$ \\
\hline Hgb-5-45 & CAYTON VALLEY HIGHLANDS & Highland area - Gb & 60.4 & $5-45$ \\
\hline Hgb-5-46 & LAKE BRITTON AREA HIGHLANDS & Highland area - Gb & $1,282.4$ & $5-46$ \\
\hline Hgb-5-47 & GOOSE VALLEY HIGHLANDS & Highland area - Gb & 73.4 & $5-47$ \\
\hline Hgb-5-48 & BURNEY CREEK VALLEY HIGHLANDS & Highland area - Gb & 187.8 & $5-48$ \\
\hline Hgb-5-49 & DRY BURNEY CREEK VALLEY HIGHLANDS & Highland area - Gb & 66.1 & $5-49$ \\
\hline Hgb-5-5 & FALL RIVER VALLEY HIGHLANDS & Highland area - Gb & $2,131.4$ & $5-5$ \\
\hline Hgb-5-50 & NORTH FORK BATTLE CREEK HIGHLANDS & Highland area - Gb & 254.9 & $5-50$ \\
\hline Hgb-5-51 & BUTTE CREEK VALLEY HIGHLANDS & Highland area - Gb & 397.1 & $5-51$ \\
\hline Hgb-5-52 & GRAYS VALLEY HIGHLANDS & Highland area - Gb & 75.0 & $5-52$ \\
\hline Hgb-5-53 & DIXIE VALLEY HIGHLANDS & Highland area - Gb & 573.7 & $5-53$ \\
\hline Hgb-5-54 & ASH VALLEY HIGHLANDS & Highland area - Gb & 220.9 & $5-54$ \\
\hline Hgb-5-56 & YELLOW CREEK VALLEY HIGHLANDS & Highland area - Gb & 78.8 & $5-56$ \\
\hline Hgb-5-57 & LAST CHANCE CREEK VALLEY HIGHLANDS & Highland area - Gb & 201.8 & $5-57$ \\
\hline Hgb-5-58 & CLOVER VALLEY HIGHLANDS & Highland area - Gb & 208.7 & $5-58$ \\
\hline Hgb-5-59 & GRIZZLY VALLEY HIGHLANDS & Highland area - Gb & 84.6 & $5-59$ \\
\hline Hgb-5-6 & REDDING AREA HIGHLANDS & Highland area - Gb & $8,237.2$ & $5-6$ \\
\hline Hgb-5-60 & HUMBUG VALLEY HIGHLANDS & Highland area - Gb & 136.0 & $5-60$ \\
\hline Hgb-5-61 & CHROME TOWN AREA HIGHLANDS & Highland area - Gb & 33.5 & $5-61$ \\
\hline Hgb-5-62 & ELK CREEK AREA HIGHLANDS & Highland area - Gb & 281.4 & $5-62$ \\
\hline Hgb-5-63 & STONYFORD TOWN AREA HIGHLANDS & Highland area - Gb & 331.5 & $5-63$ \\
\hline Hgb-5-64 & BEAR VALLEY HIGHLANDS & Highland area - Gb & 92.9 & $5-64$ \\
\hline Hgb-5-65 & LITTLE INDIAN VALLEY HIGHLANDS & Highland area - Gb & 94.9 & $5-65$ \\
\hline Hgb-5-66 & CLEAR LAKE CACHE FORMATION HIGHLANDS & Highland area - Gb & 122.9 & $5-66$ \\
\hline Hgb-5-68 & POPE VALLEY HIGHLANDS & Highland area - Gb & 159.4 & $5-68$ \\
\hline Hgb-5-69 & YOSEMITE VALLEY HIGHLANDS & Highland area - Gb & 889.0 & $5-69$ \\
\hline Hgb-5-7 & LAKE ALMANOR VALLEY HIGHLANDS & Highland area - Gb & 403.0 & $5-7$ \\
\hline Hgb-5-70 & LOS BANOS CREEK VALLEY HIGHLANDS & Highland area - Gb & 307.4 & $5-70$ \\
\hline Hgb-5-71 & VALLECITOS CREEK VALLEY HIGHLANDS & Highland area - Gb & 205.4 & $5-71$ \\
\hline Hgb-5-8 & MOUNTAIN MEADOWS VALLEY HIGHLANDS & Highland area - Gb & 211.7 & $5-8$ \\
\hline Hgb-5-82 & CUDDY CANYON VALLEY HIGHLANDS & Highland area - Gb & 37.3 & $5-82$ \\
\hline Hgb-5-83 & CUDDY RANCH AREA HIGHLANDS & Highland area - Gb & 26.7 & $5-83$ \\
\hline Hgb-5-84 & CUDDY VALLEY HIGHLANDS & Highland area - Gb & 30.2 & $5-84$ \\
\hline Hgb-5-85 & MIL POTRERO AREA HIGHLANDS & Highland area - Gb & 25.6 & $5-85$ \\
\hline Hgb-5-86 & JOSEPH CREEK HIGHLANDS & Highland area - Gb & 124.0 & $5-86$ \\
\hline Hgb-5-87 & MIDDLE FORK FEATHER RIVER HIGHLANDS & Highland area - Gb & 141.4 & $5-87$ \\
\hline Hgb-5-88 & STONY GORGE RESERVOIR HIGHLANDS & Highland area - Gb & 280.7 & $5-88$ \\
\hline Hgb-5-89 & SQUAW FLAT HIGHLANDS & Highland area - Gb & 29.8 & $5-89$ \\
\hline
\end{tabular}


Table 1. California Groundwater Units.-Continued

[GU ID is the unique groundwater unit identifier. GU name is the groundwater unit name. GU names in uppercase are the same names as those used in CDWR 2002 groundwater basin map publication except for the word "HIGHLANDS" when appended. GU type identifies one of three types of groundwater units: Groundwater basin, Highland area - Gb (groundwater-basin-based highland), or Highland area - Province (province-based highland). Related Gb references the basin-ID or subbasin-ID in the CDWR 2002 groundwater basin map publication. CDWR, California Department of Water Resources; km², square kilometer; —, no data]

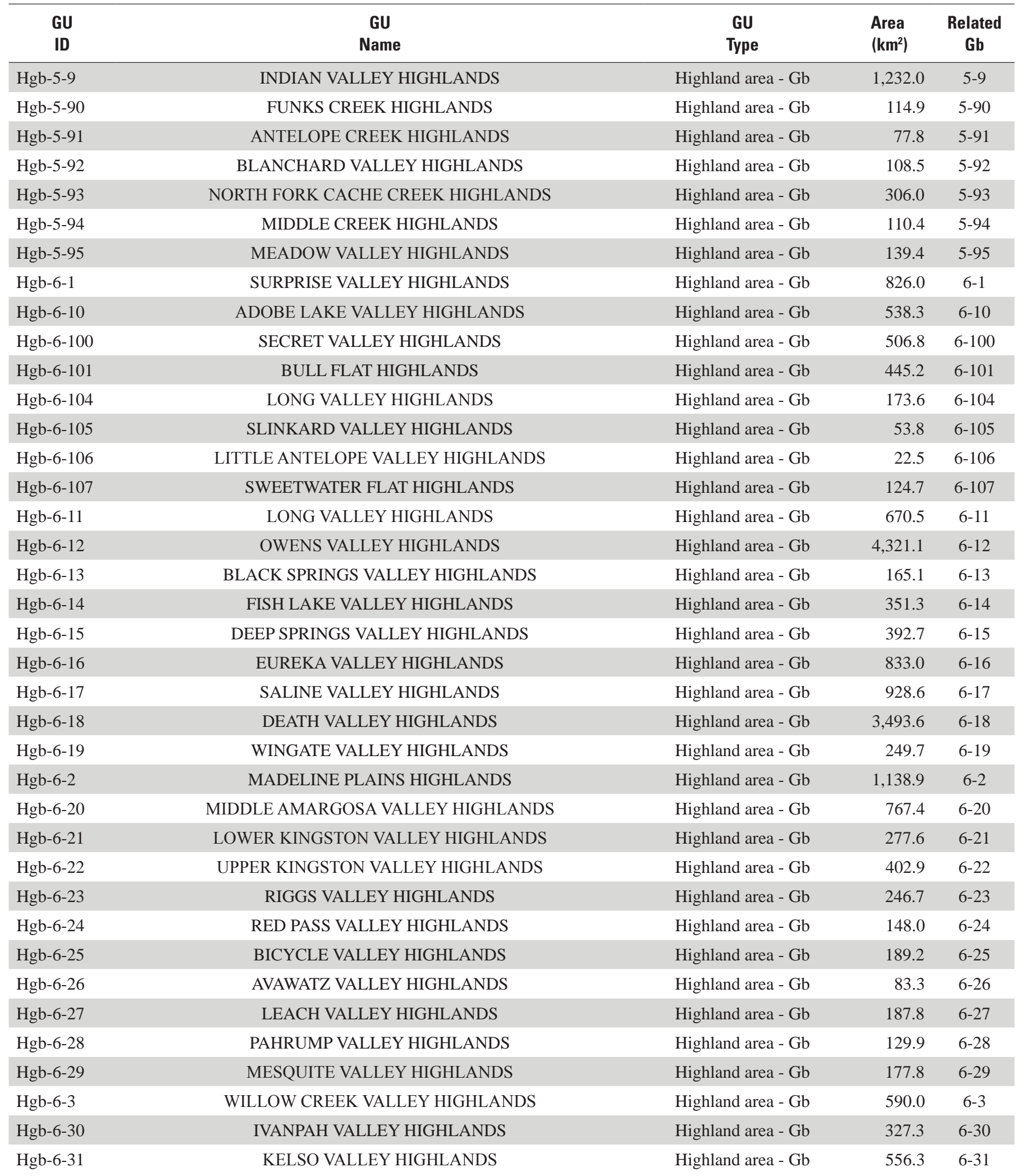


Table 1. California Groundwater Units.-Continued

[GU ID is the unique groundwater unit identifier. GU name is the groundwater unit name. GU names in uppercase are the same names as those used in CDWR 2002 groundwater basin map publication except for the word "HIGHLANDS" when appended. GU type identifies one of three types of groundwater units: Groundwater basin, Highland area - Gb (groundwater-basin-based highland), or Highland area - Province (province-based highland). Related Gb references the basin-ID or subbasin-ID in the CDWR 2002 groundwater basin map publication. CDWR, California Department of Water Resources; km², square kilometer; —, no data]

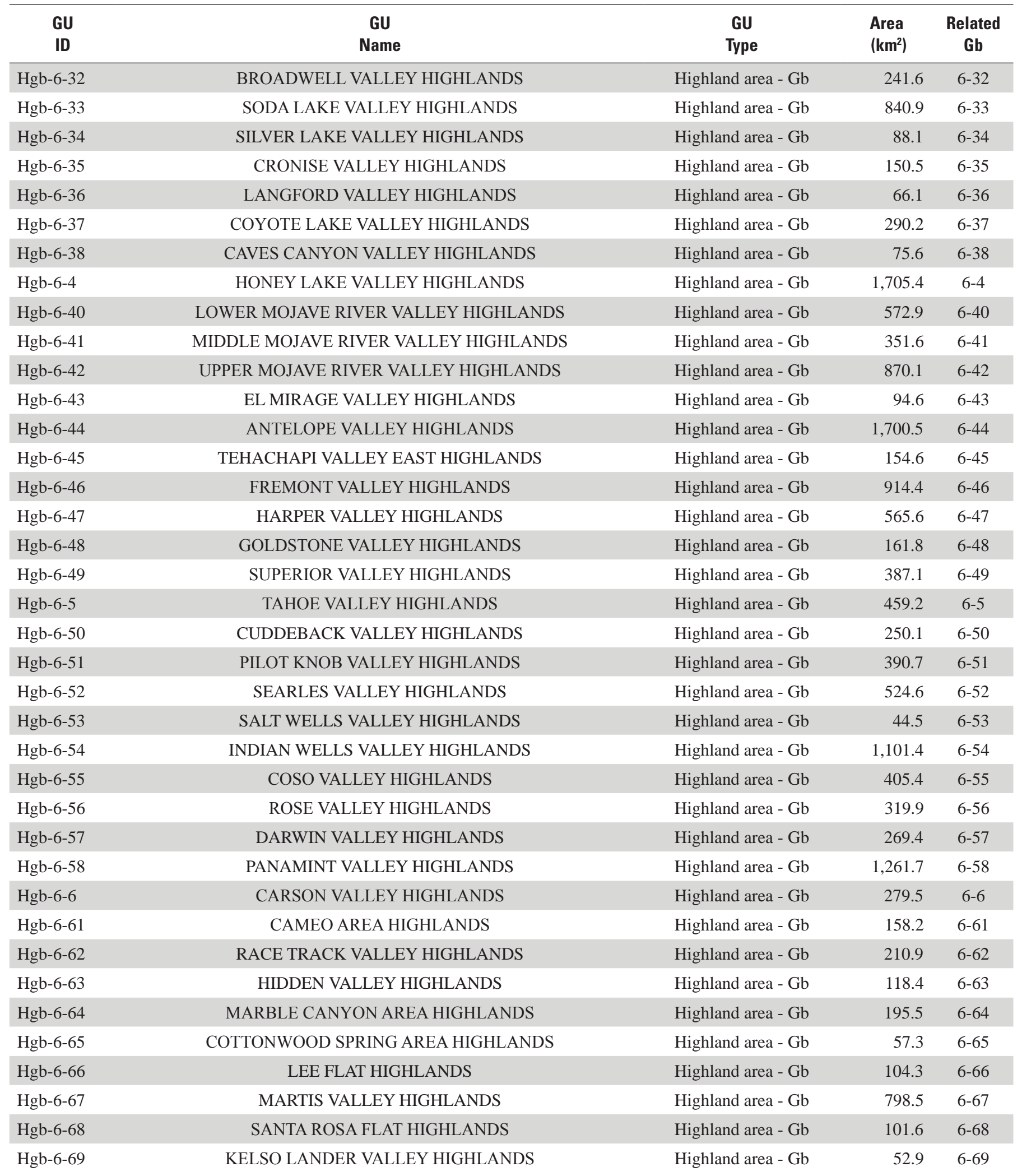


Table 1. California Groundwater Units.-Continued

[GU ID is the unique groundwater unit identifier. GU name is the groundwater unit name. GU names in uppercase are the same names as those used in CDWR 2002 groundwater basin map publication except for the word "HIGHLANDS" when appended. GU type identifies one of three types of groundwater units: Groundwater basin, Highland area - Gb (groundwater-basin-based highland), or Highland area - Province (province-based highland). Related Gb references the basin-ID or subbasin-ID in the CDWR 2002 groundwater basin map publication. CDWR, California Department of Water Resources; km², square kilometer; -, no data]

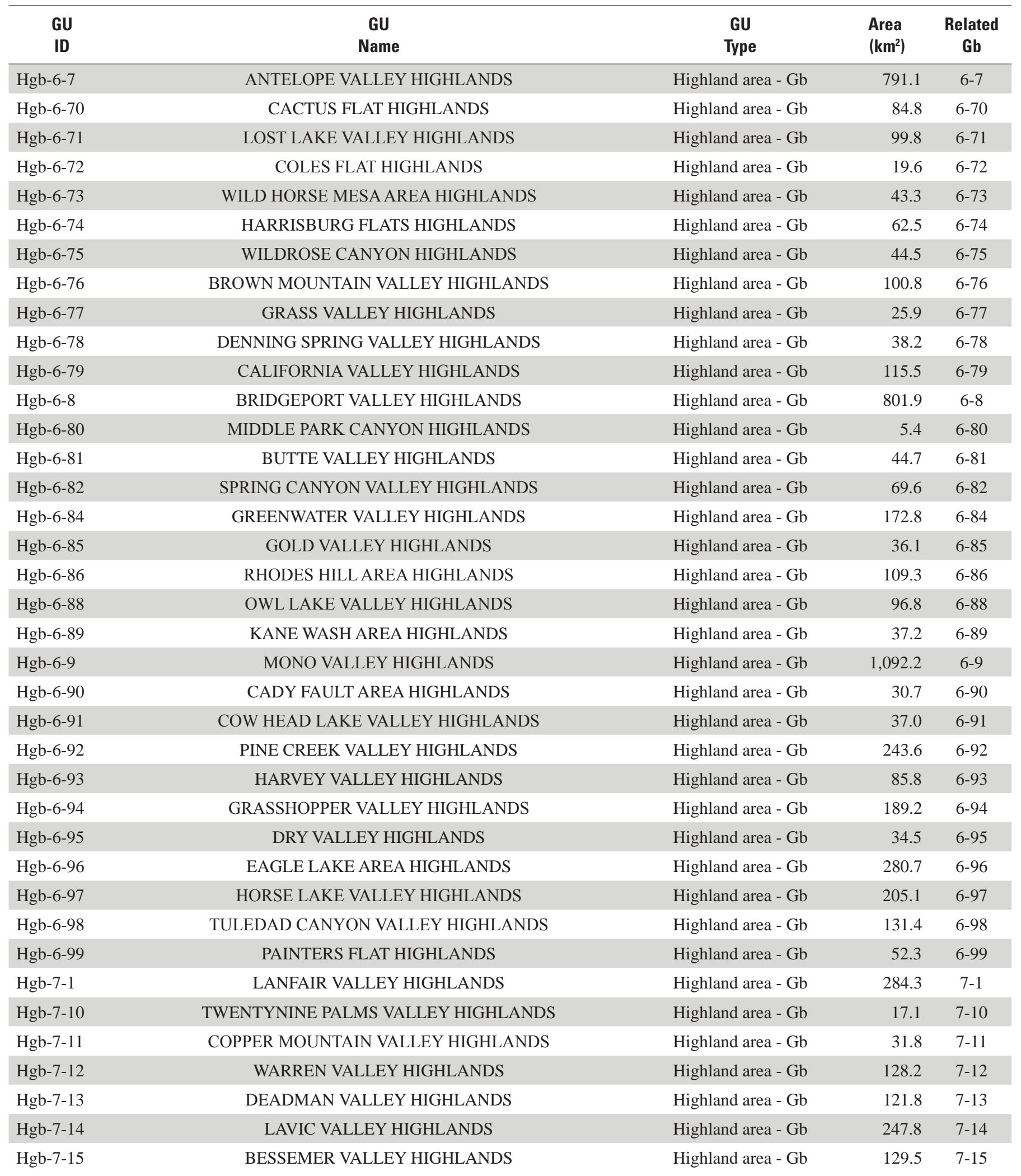


Table 1. California Groundwater Units.-Continued

[GU ID is the unique groundwater unit identifier. GU name is the groundwater unit name. GU names in uppercase are the same names as those used in CDWR 2002 groundwater basin map publication except for the word "HIGHLANDS" when appended. GU type identifies one of three types of groundwater units: Groundwater basin, Highland area - Gb (groundwater-basin-based highland), or Highland area - Province (province-based highland). Related Gb references the basin-ID or subbasin-ID in the CDWR 2002 groundwater basin map publication. CDWR, California Department of Water Resources; km², square kilometer; —, no data]

\begin{tabular}{|c|c|c|c|c|}
\hline $\begin{array}{l}\text { GU } \\
\text { ID }\end{array}$ & $\begin{array}{l}\text { GU } \\
\text { Name }\end{array}$ & $\begin{array}{l}\text { GU } \\
\text { Type }\end{array}$ & $\begin{array}{l}\text { Area } \\
\left(\mathbf{k m}^{2}\right)\end{array}$ & $\begin{array}{c}\text { Related } \\
\text { Gb }\end{array}$ \\
\hline Hgb-7-16 & AMES VALLEY HIGHLANDS & Highland area - Gb & 313.6 & $7-16$ \\
\hline Hgb-7-17 & MEANS VALLEY HIGHLANDS & Highland area - Gb & 29.9 & $7-17$ \\
\hline Hgb-7-18 & JOHNSON VALLEY HIGHLANDS & Highland area - Gb & 259.1 & $7-18$ \\
\hline Hgb-7-19 & LUCERNE VALLEY HIGHLANDS & Highland area - Gb & 282.0 & $7-19$ \\
\hline Hgb-7-2 & FENNER VALLEY HIGHLANDS & Highland area - Gb & 751.4 & $7-2$ \\
\hline Hgb-7-20 & MORONGO VALLEY HIGHLANDS & Highland area - Gb & 142.8 & $7-20$ \\
\hline Hgb-7-21 & COACHELLA VALLEY HIGHLANDS & Highland area - Gb & $2,159.9$ & $7-21$ \\
\hline Hgb-7-22 & WEST SALTON SEA HIGHLANDS & Highland area - Gb & 414.5 & $7-22$ \\
\hline Hgb-7-24 & BORREGO VALLEY HIGHLANDS & Highland area - Gb & 384.9 & $7-24$ \\
\hline Hgb-7-25 & OCOTILLO-CLARK VALLEY HIGHLANDS & Highland area - Gb & 433.5 & $7-25$ \\
\hline Hgb-7-26 & TERWILLIGER VALLEY HIGHLANDS & Highland area - Gb & 19.9 & $7-26$ \\
\hline Hgb-7-27 & SAN FELIPE VALLEY HIGHLANDS & Highland area - Gb & 169.9 & $7-27$ \\
\hline Hgb-7-28 & VALLECITO-CARRIZO VALLEY HIGHLANDS & Highland area - Gb & 568.0 & $7-28$ \\
\hline Hgb-7-29 & COYOTE WELLS VALLEY HIGHLANDS & Highland area - Gb & 189.0 & $7-29$ \\
\hline Hgb-7-3 & WARD VALLEY HIGHLANDS & Highland area - Gb & 680.9 & $7-3$ \\
\hline Hgb-7-30 & IMPERIAL VALLEY HIGHLANDS & Highland area - Gb & 204.1 & $7-30$ \\
\hline Hgb-7-31 & OROCOPIA VALLEY HIGHLANDS & Highland area - Gb & 306.6 & $7-31$ \\
\hline Hgb-7-32 & CHOCOLATE VALLEY HIGHLANDS & Highland area - Gb & 286.3 & $7-32$ \\
\hline Hgb-7-33 & EAST SALTON SEA HIGHLANDS & Highland area - Gb & 262.7 & 7-33 \\
\hline Hgb-7-34 & AMOS VALLEY HIGHLANDS & Highland area - Gb & 184.2 & $7-34$ \\
\hline Hgb-7-35 & OGILBY VALLEY HIGHLANDS & Highland area - Gb & 110.0 & $7-35$ \\
\hline Hgb-7-36 & YUMA VALLEY HIGHLANDS & Highland area - Gb & 194.3 & $7-36$ \\
\hline Hgb-7-37 & ARROYO SECO VALLEY HIGHLANDS & Highland area - Gb & 420.5 & $7-37$ \\
\hline Hgb-7-38 & PALO VERDE VALLEY HIGHLANDS & Highland area - Gb & 50.5 & 7-38 \\
\hline Hgb-7-39 & PALO VERDE MESA HIGHLANDS & Highland area - Gb & 240.1 & $7-39$ \\
\hline Hgb-7-4 & RICE VALLEY HIGHLANDS & Highland area - Gb & 204.4 & $7-4$ \\
\hline Hgb-7-40 & QUIEN SABE POINT VALLEY HIGHLANDS & Highland area - Gb & 98.8 & $7-40$ \\
\hline Hgb-7-41 & CALZONA VALLEY HIGHLANDS & Highland area - Gb & 137.9 & $7-41$ \\
\hline Hgb-7-42 & VIDAL VALLEY HIGHLANDS & Highland area - Gb & 176.2 & $7-42$ \\
\hline Hgb-7-43 & CHEMEHUEVI VALLEY HIGHLANDS & Highland area - Gb & 665.6 & $7-43$ \\
\hline Hgb-7-44 & NEEDLES VALLEY HIGHLANDS & Highland area - Gb & 393.0 & $7-44$ \\
\hline Hgb-7-45 & PIUTE VALLEY HIGHLANDS & Highland area - Gb & 343.3 & $7-45$ \\
\hline Hgb-7-46 & CANEBRAKE VALLEY HIGHLANDS & Highland area - Gb & 58.9 & $7-46$ \\
\hline Hgb-7-47 & JACUMBA VALLEY HIGHLANDS & Highland area - Gb & 38.8 & $7-47$ \\
\hline Hgb-7-48 & HELENDALE FAULT VALLEY HIGHLANDS & Highland area - Gb & 39.6 & $7-48$ \\
\hline Hgb-7-49 & PIPES CANYON FAULT VALLEY HIGHLANDS & Highland area - Gb & 56.3 & $7-49$ \\
\hline Hgb-7-5 & CHUCKWALLA VALLEY HIGHLANDS & Highland area - Gb & 909.9 & $7-5$ \\
\hline Hgb-7-50 & IRON RIDGE AREA HIGHLANDS & Highland area - Gb & 18.3 & $7-50$ \\
\hline
\end{tabular}


Table 1. California Groundwater Units.-Continued

[GU ID is the unique groundwater unit identifier. GU name is the groundwater unit name. GU names in uppercase are the same names as those used in CDWR 2002 groundwater basin map publication except for the word "HIGHLANDS" when appended. GU type identifies one of three types of groundwater units: Groundwater basin, Highland area - Gb (groundwater-basin-based highland), or Highland area - Province (province-based highland). Related Gb references the basin-ID or subbasin-ID in the CDWR 2002 groundwater basin map publication. CDWR, California Department of Water Resources; km², square kilometer; —, no data]

\begin{tabular}{|c|c|c|c|c|}
\hline $\begin{array}{l}\text { GU } \\
\text { ID }\end{array}$ & $\begin{array}{c}\text { GU } \\
\text { Name }\end{array}$ & $\begin{array}{l}\text { GU } \\
\text { Type }\end{array}$ & $\begin{array}{l}\text { Area } \\
\left(\mathbf{k m}^{2}\right)\end{array}$ & $\begin{array}{c}\text { Related } \\
\text { Gb }\end{array}$ \\
\hline Hgb-7-51 & LOST HORSE VALLEY HIGHLANDS & Highland area - Gb & 114.4 & $7-51$ \\
\hline Hgb-7-52 & PLEASANT VALLEY HIGHLANDS & Highland area - Gb & 87.7 & $7-52$ \\
\hline Hgb-7-53 & HEXIE MOUNTAIN AREA HIGHLANDS & Highland area - Gb & 30.1 & $7-53$ \\
\hline Hgb-7-54 & BUCK RIDGE FAULT VALLEY HIGHLANDS & Highland area - Gb & 52.1 & $7-54$ \\
\hline Hgb-7-55 & COLLINS VALLEY HIGHLANDS & Highland area - Gb & 256.8 & $7-55$ \\
\hline Hgb-7-56 & YAQUI WELL AREA HIGHLANDS & Highland area - Gb & 179.9 & $7-56$ \\
\hline Hgb-7-59 & MASON VALLEY HIGHLANDS & Highland area - Gb & 75.2 & 7-59 \\
\hline Hgb-7-6 & PINTO VALLEY HIGHLANDS & Highland area - Gb & 785.7 & $7-6$ \\
\hline Hgb-7-61 & DAVIES VALLEY HIGHLANDS & Highland area - Gb & 31.2 & $7-61$ \\
\hline Hgb-7-62 & JOSHUA TREE HIGHLANDS & Highland area - Gb & 177.5 & $7-62$ \\
\hline Hgb-7-63 & VANDEVENTER FLAT HIGHLANDS & Highland area - Gb & 33.5 & $7-63$ \\
\hline Hgb-7-7 & CADIZ VALLEY HIGHLANDS & Highland area - Gb & 386.9 & $7-7$ \\
\hline Hgb-7-8 & BRISTOL VALLEY HIGHLANDS & Highland area - Gb & 879.4 & $7-8$ \\
\hline Hgb-7-9 & DALE VALLEY HIGHLANDS & Highland area - Gb & 451.6 & $7-9$ \\
\hline Hgb-8-1 & COASTAL PLAIN OF ORANGE COUNTY HIGHLANDS & Highland area - Gb & 563.4 & 8-1 \\
\hline Hgb-8-2 & UPPER SANTA ANA VALLEY HIGHLANDS & Highland area - Gb & $1,333.6$ & $8-2$ \\
\hline Hgb-8-4 & ELSINORE HIGHLANDS & Highland area - Gb & 278.9 & $8-4$ \\
\hline Hgb-8-5 & SAN JACINTO HIGHLANDS & Highland area - Gb & 833.8 & $8-5$ \\
\hline Hgb-8-6 & HEMET LAKE VALLEY HIGHLANDS & Highland area - Gb & 115.0 & 8-6 \\
\hline Hgb-8-7 & BIG MEADOWS VALLEY HIGHLANDS & Highland area - Gb & 125.6 & $8-7$ \\
\hline Hgb-8-8 & SEVEN OAKS VALLEY HIGHLANDS & Highland area - Gb & 32.5 & $8-8$ \\
\hline Hgb-8-9 & BEAR VALLEY HIGHLANDS & Highland area - Gb & 119.8 & $8-9$ \\
\hline Hgb-9-1 & SAN JUAN VALLEY HIGHLANDS & Highland area - Gb & 404.0 & $9-1$ \\
\hline Hgb-9-10 & SAN PASQUAL VALLEY HIGHLANDS & Highland area - Gb & 236.0 & $9-10$ \\
\hline Hgb-9-11 & SANTA MARIA VALLEY HIGHLANDS & Highland area - Gb & 103.5 & $9-11$ \\
\hline Hgb-9-12 & SAN DIEGUITO CREEK HIGHLANDS & Highland area - Gb & 184.4 & $9-12$ \\
\hline Hgb-9-13 & POWAY VALLEY HIGHLANDS & Highland area - Gb & 76.9 & $9-13$ \\
\hline Hgb-9-14 & MISSION VALLEY HIGHLANDS & Highland area - Gb & 145.0 & $9-14$ \\
\hline Hgb-9-15 & SAN DIEGO RIVER VALLEY HIGHLANDS & Highland area - Gb & 977.8 & $9-15$ \\
\hline Hgb-9-16 & EL CAJON VALLEY HIGHLANDS & Highland area - Gb & 29.3 & $9-16$ \\
\hline Hgb-9-17 & SWEETWATER VALLEY HIGHLANDS & Highland area - Gb & 557.8 & $9-17$ \\
\hline Hgb-9-18 & OTAY VALLEY HIGHLANDS & Highland area - Gb & 363.3 & $9-18$ \\
\hline Hgb-9-19 & TIA JUANA HIGHLANDS & Highland area - Gb & 12.4 & $9-19$ \\
\hline Hgb-9-2 & SAN MATEO VALLEY HIGHLANDS & Highland area - Gb & 334.9 & $9-2$ \\
\hline Hgb-9-22 & BATIQUITOS LAGOON VALLEY HIGHLANDS & Highland area - Gb & 54.7 & $9-22$ \\
\hline Hgb-9-23 & SAN ELIJO VALLEY HIGHLANDS & Highland area - Gb & 75.6 & $9-23$ \\
\hline Hgb-9-24 & PAMO VALLEY HIGHLANDS & Highland area - Gb & 282.7 & $9-24$ \\
\hline Hgb-9-25 & RANCHITA TOWN AREA HIGHLANDS & Highland area - Gb & 17.0 & $9-25$ \\
\hline
\end{tabular}


Table 1. California Groundwater Units.-Continued

[GU ID is the unique groundwater unit identifier. GU name is the groundwater unit name. GU names in uppercase are the same names as those used in CDWR 2002 groundwater basin map publication except for the word "HIGHLANDS" when appended. GU type identifies one of three types of groundwater units: Groundwater basin, Highland area - Gb (groundwater-basin-based highland), or Highland area - Province (province-based highland). Related Gb references the basin-ID or subbasin-ID in the CDWR 2002 groundwater basin map publication. CDWR, California Department of Water Resources; km², square kilometer; —, no data]

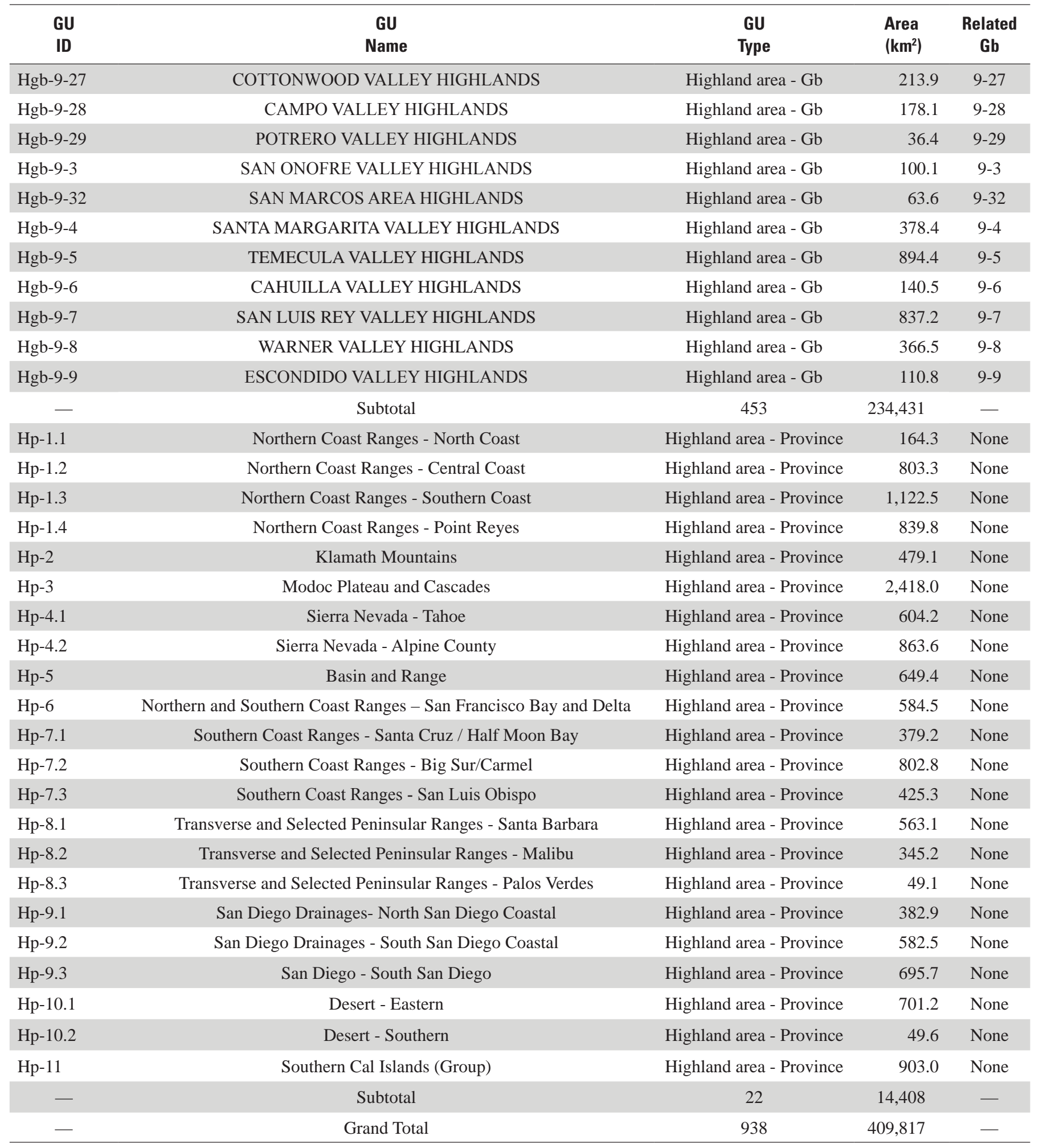




\section{Appendix: Highland Geoprocessing Tool}

The Highland geoprocessing tool was developed to assist with the automated delineation of groundwater basin highland areas. The tool was written for use in Esri's ArcGIS 10.1 software. The following is the documentation for the tool. The tool interface can be seen in figure A1, and the ModelBuilder schematic is displayed in figure A2. Two input datasets are required for the tool to operate: a flow-direction grid and a polygon feature class of groundwater basin boundaries.

The Highland tool selects the first basin in the groundwater basin input file and then temporarily codes all the other basins to a value of "1000." The tool uses the newly changed basin values to recode the cell values of the flowdirection grid to "1000" for these non-participating basins. This will effectively stop flow from moving through these reclassified basins. The net effect is a localized flow-direction grid for each selected basin that excludes any flow that will be captured by an upslope basin. Next, the tool uses Esri's Watershed tool to delineate a single drainage basin based upon this newly created, custom flow-direction grid, effectively creating a highland area for the groundwater basin. The Highland tool then selects the next basin and restarts the process. The tool will iterate through the entire input dataset until a highland has been delineated for each groundwater basin for which one should exist. Some basins may be completely surrounded by other groundwater basins, and therefore, no highland area will be created. Once highland areas have been created for all appropriate basins, they can be merged together into a single geodataset in a separate process outside of the Highland tool. The tool does not delineate province-based highland areas.

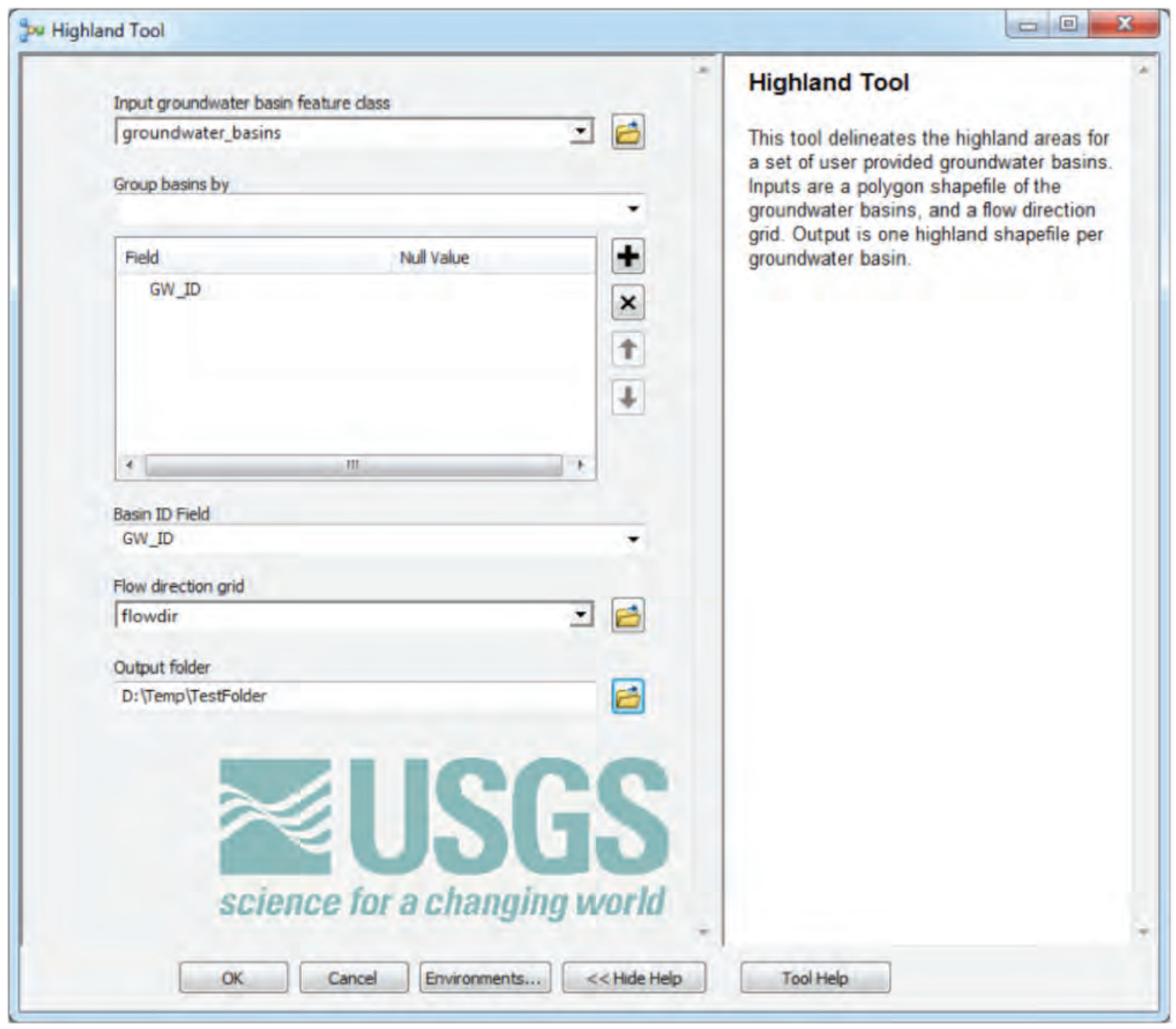

Figure A1. Example of Highland tool interface within ArcGIS. 


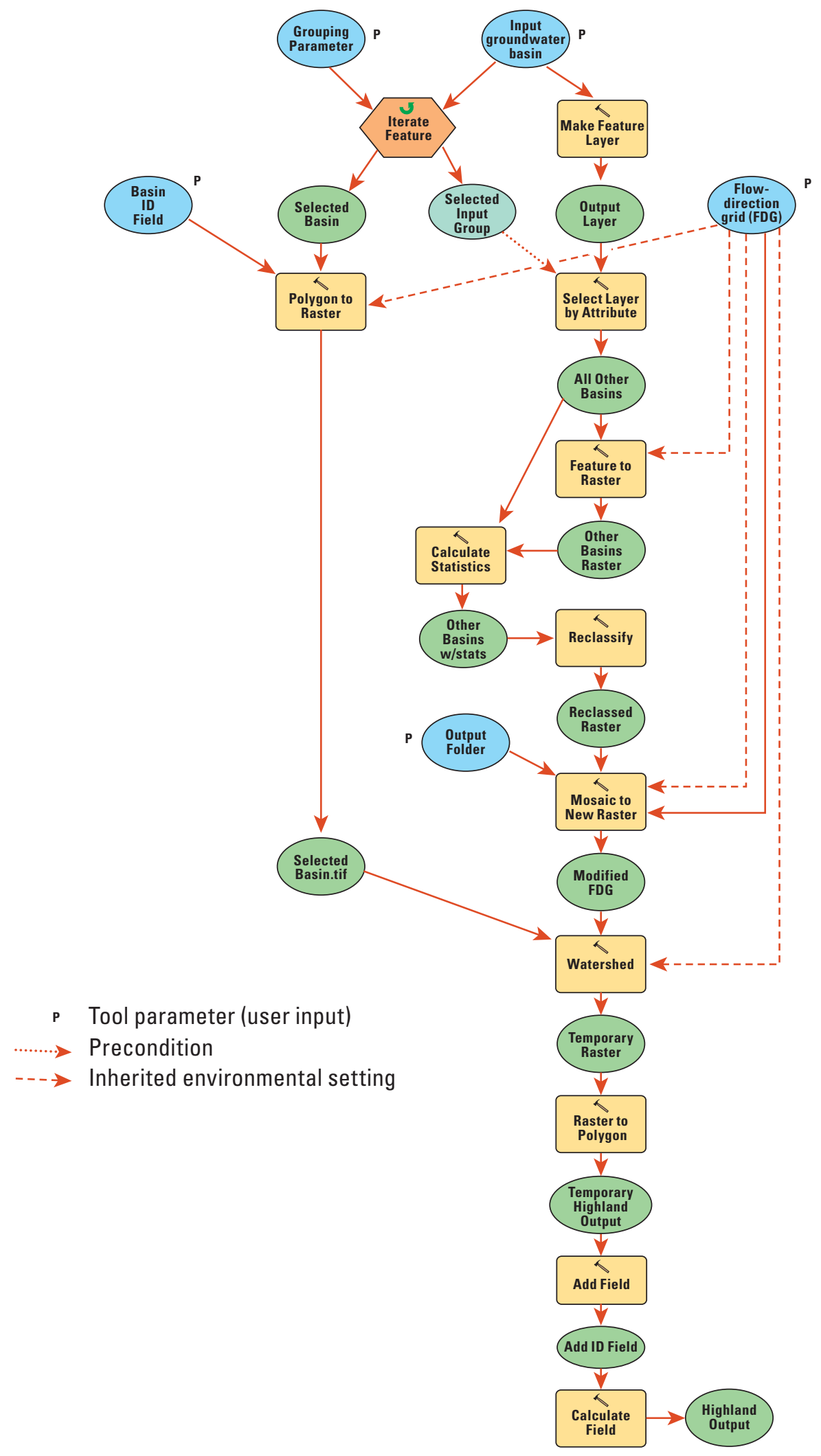

Figure A2. ArcGIS ModelBuilder (Esri) schematic of the Highland tool. 
Publishing support provided by the U.S. Geological Survey

Science Publishing Network, Sacramento, Tacoma, and Raleigh Publishing Service Centers

For more information concerning the research in this report, contact the

Director, California Water Science Center

U.S. Geological Survey

6000 J Street, Placer Hall

Sacramento, California 95819

http://ca.water.usgs.gov 


\section{$\frac{\mathbb{2}}{\mathscr{3}}$}

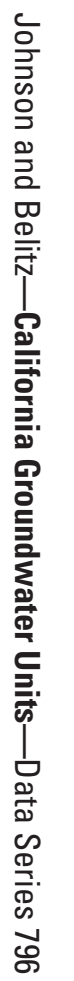

